\title{
General formulas for capacity of classical-quantum channels
}

\author{
Masahito Hayashi $^{*} \quad$ Hiroshi Nagaoka ${ }^{\dagger}$
}

\begin{abstract}
The capacity of a classical-quantum channel (or in other words the classical capacity of a quantum channel) is considered in the most general setting, where no structural assumptions such as the stationary memoryless property are made on a channel. A capacity formula as well as a characterization of the strong converse property is given just in parallel with the corresponding classical results of Verdú-Han which are based on the so-called informationspectrum method. The general results are applied to the stationary memoryless case with or without cost constraint on inputs, whereby a deep relation between the channel coding theory and the hypothesis testing for two quantum states is elucidated.
\end{abstract}

\section{Keywords}

Quantum channel coding, Information spectrum, Classical-quantum channel, Classical capacity of a quantum channel, Cost constraint

\section{Introduction}

The channel coding theorem for a stationary and memoryless ${ }^{1}$ (classical-)quantum channel has been established by combining the direct part shown by Holevo [1] and Schumacher-Westmoreland 2] with the (weak) converse part which goes back to 1970's works by Holevo 3, 4. This theorem is undoubtedly a landmark in the history of quantum information theory. At the same time, however, we should not forget that stationary memoryless channels are not the only class of quantum channels.

\footnotetext{
*Laboratory for Mathematical Neuroscience, Brain Science Institute, RIKEN, 2-1 Hirosawa, Wako, Saitama, 351-0198, Japan. (e-mail: masahito@brain.riken.go.jp)

${ }^{\dagger}$ Graduate School of Information Systems, University of Electro-Communications. 1-5-1, Chouhugaoka, Chouhu-shi, Tokyo, 182-8585, Japan. (e-mail: nagaoka@is.uec.ac.jp)

${ }^{1}$ Throughout the paper, a stationary memoryless channel without using entangled input states is simply referred to as a stationary memoryless channel; see Remark 13.
} 
It is indeed natural to think that many channels appearing in nature are neither stationary nor memoryless even in the approximate sense.

In the classical information theory, a capacity formula for the most general setting was given by Verdú and Han [5], based on the so-called information-spectrum method 6]. We show in this paper that a similar approach is applicable to yield some general formulas for the capacity of a classical-quantum channel (or in other words the classical-capacity of a quantum channel) and related notions.

Let us take a brief look at the general feature of the information-spectrum method in the classical information theory. One of the main subjects of the information theory is to characterize asymptotic optimalities of various types of coding problems by entropy-like information quantities. In the information-spectrum method, a coding problem is treated in the most general setting, without assuming any structural assumptions such as the stationary memoryless property, and the asymptotic optimality is characterized by a limiting expression on information spectra (i.e., asymptotic behaviors of logarithmic likelihoods). Since the asymptotic optimization of coding is essentially solved in this characterization, rewriting the information-spectrum quantity to an entropy-like quantity for a specific situation is mostly a direct consequence of a limiting theorem in the probability theory such as the law of large numbers, the Shannon-McMillan-Breiman theorem, ergodic theorems, large deviation theorems, etc. Such a framework brings not only generality but also transparency of mathematical arguments. Indeed, we are often led to simplification of a proof of an existing coding theorem by investigating it from the information-spectrum viewpoint.

Turning to the quantum information theory, in spite of the recent remarkable progress of the field we often see that mathematical arguments to prove theorems are neither so transparent nor unified as in the classical theory. For instance, the original proof of the direct part of quantum channel coding theorem [1, 2] is rather complicated so that it is not easy to grasp the essence of the argument(; see [7 for a different proof). Extending the information-spectrum method to the quantum case is an attractive subject which brings a hope that proofs will be simplified and, more importantly, that both the optimality of coding systems and the limiting law governing quantum stochastic situations will be provided with transparent and comprehensive understanding.

In this paper, we pursue this subject for the quantum channel coding problem, whereby the quantum analogue of Verudú-Han's general formula is obtained. In addition, the formula is applied to the stationary memoryless case to yield a new proof of the quantum channel coding theorem. It should be noted here that, in both of derivation of the general formula and application to the stationary memoryless case to get a nonasymptotic expression, there arise several mathematical difficulties to which the corresponding classical arguments are not immediately applicable. The difficulties in deriving the general formula are overcome by using the quantum Neyman-Pearson lemma [8, 9, 10] and a novel operator inequality (Lemma 2), while those in rewriting the formula to the known form in the stationary memoryless case are coped with by invoking the asymptotic theory of hypothesis testing for two quan- 
tum states [11, 12, 10] (; see the references of [10] for related results) as a kind of substitute of the weak law of large numbers. In particular, the inequality of Lemma 2 is expected to play a key role in analyzing a measurement of the square root type in general; actually it drastically simplifies the original proof of [1, 2] as mentioned in Remark [19.

Historically, the present work is preceded by Ogawa's proof [13] of the direct part of the quantum channel coding theorem, with an improved and simplified version being found in [14, which was actually the first remarkable result of the informationspectrum approach to the quantum channel coding problem and elucidated the close relation between the channel coding and the hypothesis testing in the quantum information theory; see Remark 9 and Remark 14. In the present paper, we clarify this relation from a more general viewpoint and make further developments to establish the information-spectrum method in the quantum channel coding theory. These attempts lead us to better understanding of the reason why the quantum relative entropy plays important roles in both of these problems.

We should emphasize, however, that the present paper is not the final goal for the information-spectrum study of quantum channel capacity. Even though a general capacity formula has been given in terms of the quantum information spectrum, the way to apply it to the stationary memoryless case shown in this paper is not so straightforward as the classical counterpart. Indeed, if our concern is restricted to proving the coding theorem for stationary memoryless channels, the information spectrum appears to be a kind of roundabout at present; see Remarks [15, 17] and 19. In order to achieve the same level of simplicity and transparency as the classical information-spectrum method and to fulfill further the above-mentioned hope for the quantum information-spectrum method, we will need to have more theoretical tools to analyze the quantum information spectrum.

The paper is organized as follows. In section 2 the notion of general classicalquantum channels is introduced and the coding problem for it is formulated. Section 3 is devoted to asserting the main theorem, which gives the general capacity formula and the characterization of strong converse property of a general channel, while the proof is given in section [5] based on some lemmas prepared in section 4. Stationary memoryless channels are treated in section 6 and section 8 , the latter of which considers cost constraint on inputs, while section $\mathbf{7}$ is devoted to revisiting the decoder introduced by Holevo-Schumacher-Westmoreland in view of comparison to our decoder used to prove the general formulas. Section 9 gives some concluding remarks.

\section{Capacity of general classical-quantum channels}

A quantum communication channel is generally composed of the following constructs; (separable) Hilbert spaces $\mathcal{H}_{1}$ and $\mathcal{H}_{2}$ which respectively represent the quantum systems of the sender's and the receiver's sides, a trace preserving CP (completely 
positive) map $\Gamma$ from the trace-class operators on $\mathcal{H}_{1}$ to those on $\mathcal{H}_{2}$ which describes the change of sent states, and a map $V: \mathcal{X} \rightarrow \mathcal{S}\left(\mathcal{H}_{1}\right)$ which represents the modulator to set the input state to $V_{x}$ according to the value of the control variable $x \in \mathcal{X}$. When our concern is restricted to sending classical messages via the channel, however, only the composite map $\Gamma \circ V: \mathcal{X} \rightarrow \mathcal{S}\left(\mathcal{H}_{2}\right)$ is relevant, and hence in the sequel we call a map $W: \mathcal{X} \ni x \mapsto W_{x} \in \mathcal{S}(\mathcal{H})$ a classical-quantum channel or simply a channel. Here $\mathcal{X}$ is an arbitrary (finite or infinite) set and $\mathcal{H}$ is an arbitrary Hilbert space. This definition corresponds to the classical one in which a channel is represented by a conditional probability $W:(x, y) \mapsto W(y \mid x)$ or equivalently by a map $W: x \mapsto W_{x}=W(\cdot \mid x)$.

Remark 1 In many papers treating the capacity of quantum memoryless channels (e.g., [1, 2, 3, 7, 15]), only the case when $\mathcal{X}$ is a finite set is considered. Even though the restriction to the finite case may be sufficient to understand the essence of most (but not all) mathematical arguments for proving the capacity theorem, there is no reason to restrict ourselves to the finite case from the standpoint that the capacity is the maximum reliable transmission rate of all possible communication systems for a given quantum channel. Indeed, a particularly important infinite case is when $\mathcal{X}=\mathcal{S}\left(\mathcal{H}_{1}\right)$ and $W$ is a trace-preserving CP map.

Remark 2 The term "classical-quantum channel" has been provided with several different meanings in the literature (cf. [16]). The present definition is similar to that of [17, although some measure-theoretic assumptions were made there on both the set $\mathcal{X}$ and the mapping $x \mapsto W_{x}$ to consider a channel in a general and unified operator-algebraic setting.

Remark 3 As was pointed out in [18, the capacity problem for a channel $W$ : $\mathcal{X} \rightarrow \mathcal{S}(\mathcal{H})$ relies only on its range $\left\{W_{x} \mid x \in \mathcal{X}\right\}$, and we can adopt the alternative definition in which an arbitrary subset of $\mathcal{S}(\mathcal{H})$ is called a channel. In other words, we can assume, if we wish, with no loss of generality that every $W$ appearing in the sequel is the identity map on a subset $\mathcal{X} \subset \mathcal{S}(\mathcal{H})$. The reason for treating a map $W$ instead of its range is mainly that it enables us to introduce more readable and natural notations.

For an arbitrary channel $W: \mathcal{X} \rightarrow \mathcal{S}(\mathcal{H})$, we call a triple $(N, \varphi, Y)$ a code for $W$ when it consists of a natural number (size) $N$, a mapping (encoding) $\varphi$ : $\{1, \ldots, N\} \rightarrow \mathcal{X}$ and a POVM (decoding) $Y=\left\{Y_{i}\right\}_{i=1}^{N}$ on $\mathcal{H}$ such that $\sum_{i} Y_{i} \leq I$, where $I-\sum_{i} Y_{i}$ corresponds to the failure of decoding, and denote the totality of such codes by $\mathfrak{C}(W)$. For a code $\Phi=(N, \varphi, Y) \in \mathfrak{C}(W)$, the code size and the average error probability are represented as

$$
\begin{gathered}
|\Phi| \stackrel{\text { def }}{=} N, \quad \text { and } \\
\mathrm{P}_{\mathrm{e}}[\Phi] \stackrel{\text { def }}{=} \frac{1}{N} \sum_{i=1}^{N}\left(1-\operatorname{Tr}\left[W_{\varphi(i)} Y_{i}\right]\right) .
\end{gathered}
$$


Now let us proceed to the asymptotic setting. Suppose that we are given a sequence $\overrightarrow{\mathcal{H}}=\left\{\mathcal{H}^{(n)}\right\}_{n=1}^{\infty}$ of Hilbert spaces and a sequence $\overrightarrow{\boldsymbol{W}}=\left\{W^{(n)}\right\}_{n=1}^{\infty}$ of channels $W^{(n)}: \mathcal{X}^{(n)} \rightarrow \mathcal{S}\left(\mathcal{H}^{(n)}\right)$. An important example is the stationary memoryless case when $\overrightarrow{\mathcal{H}}$ and $\overrightarrow{\boldsymbol{W}}$ are defined from a Hilbert space $\mathcal{H}$ and a channel $W: \mathcal{X} \rightarrow \mathcal{S}(\mathcal{H})$ as $\mathcal{H}^{(n)}=\mathcal{H}^{\otimes n}, \mathcal{X}^{(n)}=\mathcal{X}^{n}$ and $W_{x^{n}}^{(n)}=W_{x_{1}} \otimes \cdots \otimes W_{x_{n}}$ for $x^{n}=\left(x_{1}, \ldots, x_{n}\right)$, which will be treated in sections 6 and 8 . Except for those sections, however, we do not make any assumptions on the mutual relations among $\left\{\mathcal{H}^{(n)}\right\},\left\{\mathcal{X}^{(n)}\right\}$ and $\left\{W^{(n)}\right\}$ for different $n$ 's. Such an extremely general setting is one of the main features of the information spectrum approach. The capacity of $\overrightarrow{\boldsymbol{W}}$ is then defined as

$$
\begin{aligned}
C(\overrightarrow{\boldsymbol{W}}) \stackrel{\text { def }}{=} \sup \left\{R \mid \exists \overrightarrow{\boldsymbol{\Phi}}=\left\{\Phi^{(n)}\right\} \in \overrightarrow{\mathfrak{C}}(\overrightarrow{\boldsymbol{W}}),\right. \\
\left.\quad \liminf _{n \rightarrow \infty} \frac{1}{n} \log \left|\Phi^{(n)}\right| \geq R \text { and } \lim _{n \rightarrow \infty} \mathrm{P}_{\mathrm{e}}\left[\Phi^{(n)}\right]=0\right\},
\end{aligned}
$$

where $\overrightarrow{\mathfrak{C}}(\overrightarrow{\boldsymbol{W}})$ denotes the totality of sequences of codes $\overrightarrow{\boldsymbol{\Phi}}=\left\{\Phi^{(n)}\right\}_{n=1}^{\infty}$ such that $\Phi^{(n)} \in \mathfrak{C}\left(W^{(n)}\right)$ for all $n$. We also introduce a 'dual' of the capacity

$$
\begin{aligned}
C^{\dagger}(\overrightarrow{\boldsymbol{W}}) \stackrel{\text { def }}{=} \inf \left\{R \mid \forall \overrightarrow{\boldsymbol{\Phi}}=\left\{\Phi^{(n)}\right\} \in \overrightarrow{\mathfrak{C}}(\overrightarrow{\boldsymbol{W}}),\right. \\
\left.\qquad \liminf _{n \rightarrow \infty} \frac{1}{n} \log \left|\Phi^{(n)}\right| \geq R \text { implies } \lim _{n \rightarrow \infty} \mathrm{P}_{\mathrm{e}}\left[\Phi^{(n)}\right]=1\right\} .
\end{aligned}
$$

Note that $C(\overrightarrow{\boldsymbol{W}}) \leq C^{\dagger}(\overrightarrow{\boldsymbol{W}})$ always holds. Following the terminology of classical information theory, we say that the strong converse holds for $\overrightarrow{\boldsymbol{W}}$ when $C(\overrightarrow{\boldsymbol{W}})=$ $C^{\dagger}(\overrightarrow{\boldsymbol{W}})$.

\section{Main results}

In this section we give general formulas for $C(\overrightarrow{\boldsymbol{W}})$ and $C^{\dagger}(\overrightarrow{\boldsymbol{W}})$ which are regarded as the quantum extensions of those for classical channel coding obtained by Verdú and Han [5]. The classical formula was given in terms of some information-spectrumtheoretic quantities, and we first need to introduce quantum analogues of these concepts along the line developed in [10].

For a self-adjoint trace-class operator $A$ with the spectral decomposition $A=$ $\sum_{i} \lambda_{i} E_{i}$, where $\left\{\lambda_{i}\right\}$ are the eigenvalues and $\left\{E_{i}\right\}$ are the orthogonal projections onto the corresponding eigenspaces, we define

$$
\{A \geq 0\} \stackrel{\text { def }}{=} \sum_{i: \lambda_{i} \geq 0} E_{i} \quad \text { and } \quad\{A>0\} \stackrel{\text { def }}{=} \sum_{i: \lambda_{i}>0} E_{i} .
$$

These are the orthogonal projections onto the direct sum of eigenspaces corresponding to nonnegative and positive eigenvalues, respectively. The projections $\{A \leq 0\}$ and $\{A<0\}$ are defined similarly. 
For any set $\mathcal{X}$, let $\mathcal{P}(\mathcal{X})$ be the totality of probability distributions on $\mathcal{X}$ with finite supports. That is, an element $P$ of $\mathcal{P}(\mathcal{X})$ is a function $\mathcal{X} \rightarrow[0,1]$ such that its $\operatorname{support} \operatorname{supp}(P) \stackrel{\text { def }}{=}\{x \mid P(x)>0\}$ is a finite set and that

$$
\sum_{x \in \mathcal{X}} P(x)=\sum_{x \in \operatorname{supp}(P)} P(x)=1 .
$$

Let the totality of sequences $\overrightarrow{\boldsymbol{P}}=\left\{P^{(n)}\right\}_{n=1}^{\infty}$ of $P^{(n)} \in \mathcal{P}\left(\mathcal{X}^{(n)}\right)$ be denoted by $\overrightarrow{\mathcal{P}}(\overrightarrow{\mathcal{X}})$, and the totality of $\overrightarrow{\boldsymbol{\sigma}}=\left\{\sigma^{(n)}\right\}_{n=1}^{\infty}$ of $\sigma^{(n)} \in \mathcal{S}(\mathcal{H})$ by $\overrightarrow{\mathcal{S}}(\overrightarrow{\mathcal{H}})$. Given $\overrightarrow{\boldsymbol{P}} \in \overrightarrow{\mathcal{P}}(\overrightarrow{\mathcal{X}})$ and $\overrightarrow{\boldsymbol{\sigma}} \in \overrightarrow{\mathcal{S}}(\overrightarrow{\mathcal{H}})$, let

$\bar{J}(\overrightarrow{\boldsymbol{P}}, \overrightarrow{\boldsymbol{\sigma}}, \overrightarrow{\boldsymbol{W}}) \stackrel{\text { def }}{=} \inf \left\{a \mid \lim _{n \rightarrow \infty} \sum_{x^{n} \in \mathcal{X}^{(n)}} P^{(n)}\left(x^{n}\right) \operatorname{Tr}\left[W_{x^{n}}^{(n)}\left\{W_{x^{n}}^{(n)}-e^{n a} \sigma^{(n)}>0\right\}\right]=0\right\}$, $\underline{J}(\overrightarrow{\boldsymbol{P}}, \overrightarrow{\boldsymbol{\sigma}}, \overrightarrow{\boldsymbol{W}}) \stackrel{\text { def }}{=} \sup \left\{a \mid \lim _{n \rightarrow \infty} \sum_{x^{n} \in \mathcal{X}^{(n)}} P^{(n)}\left(x^{n}\right) \operatorname{Tr}\left[W_{x^{n}}^{(n)}\left\{W_{x^{n}}^{(n)}-e^{n a} \sigma^{(n)}>0\right\}\right]=1\right\}$,

and

$$
\begin{aligned}
& \bar{I}(\overrightarrow{\boldsymbol{P}}, \overrightarrow{\boldsymbol{W}}) \stackrel{\text { def }}{=} \bar{J}\left(\overrightarrow{\boldsymbol{P}}, \overrightarrow{\boldsymbol{W}}_{\overrightarrow{\boldsymbol{P}}}, \overrightarrow{\boldsymbol{W}}\right), \\
& \underline{I}(\overrightarrow{\boldsymbol{P}}, \overrightarrow{\boldsymbol{W}}) \stackrel{\text { def }}{=} \underline{J}\left(\overrightarrow{\boldsymbol{P}}, \overrightarrow{\boldsymbol{W}}_{\overrightarrow{\boldsymbol{P}}}, \overrightarrow{\boldsymbol{W}}\right),
\end{aligned}
$$

where $\overrightarrow{\boldsymbol{W}}_{\overrightarrow{\boldsymbol{P}}}$ denotes the sequence $\left\{W_{P^{(n)}}^{(n)}\right\}_{n=1}^{\infty}$ of

$$
W_{P^{(n)}}^{(n)} \stackrel{\text { def }}{=} \sum_{x^{n} \in \mathcal{X}^{(n)}} P^{(n)}\left(x^{n}\right) W_{x^{n}}^{(n)} \in \mathcal{S}\left(\mathcal{H}^{(n)}\right) .
$$

Note that $\bar{I}(\overrightarrow{\boldsymbol{P}}, \overrightarrow{\boldsymbol{W}})$ and $\underline{I}(\overrightarrow{\boldsymbol{P}}, \overrightarrow{\boldsymbol{W}})$ are quantum analogues of the spectral sup- and inf-information rates $(\underline{5})$ :

$$
\begin{aligned}
& \bar{I}(\mathbf{X} ; \mathbf{Y}) \stackrel{\text { def }}{=} \mathrm{p}-\limsup _{n \rightarrow \infty} \frac{1}{n} \log \frac{W^{(n)}\left(Y^{(n)} \mid X^{(n)}\right)}{P_{Y^{(n)}}\left(Y^{(n)}\right)}, \\
& \underline{I}(\mathbf{X} ; \mathbf{Y}) \stackrel{\text { def }}{=} \mathrm{p}-\liminf _{n \rightarrow \infty} \frac{1}{n} \log \frac{W^{(n)}\left(Y^{(n)} \mid X^{(n)}\right)}{P_{Y^{(n)}}\left(Y^{(n)}\right)}
\end{aligned}
$$

where $\mathbf{Y}=\left\{Y^{(n)}\right\}$ is supposed to be the sequence of random variables obtained as the outputs of channels $\mathbf{W}=\left\{W^{(n)}\right\}$ for a sequence of input random variables $\mathbf{X}=\left\{X^{(n)}\right\}$.

Remark 4 The projection $\left\{W_{x^{n}}^{(n)}-e^{n a} \sigma^{(n)}>0\right\}$ in the definitions above can be replaced with $\left\{W_{x^{n}}^{(n)}-e^{n a} \sigma^{(n)} \geq 0\right\}$ or, more generally, with an arbitrary self-adjoint operator $S$ satisfying

$$
\left\{W_{x^{n}}^{(n)}-e^{n a} \sigma^{(n)}>0\right\} \leq S \leq\left\{W_{x^{n}}^{(n)}-e^{n a} \sigma^{(n)} \geq 0\right\} .
$$

This ambiguity does not influence the definitions of the above quantities; see [10]. 
Now we have the following theorem.

\section{Theorem 1}

$$
\begin{aligned}
C(\overrightarrow{\boldsymbol{W}}) & =\max _{\overrightarrow{\boldsymbol{P}} \in \overrightarrow{\mathcal{P}}(\overrightarrow{\mathcal{X}})} I(\overrightarrow{\boldsymbol{P}}, \overrightarrow{\boldsymbol{W}}) \\
& =\max _{\overrightarrow{\boldsymbol{P}} \in \overrightarrow{\mathcal{P}}(\overrightarrow{\mathcal{X}})} \min _{\overrightarrow{\boldsymbol{\sigma}} \in \overrightarrow{\mathcal{S}}(\overrightarrow{\mathcal{H}})} \underline{J}(\overrightarrow{\boldsymbol{P}}, \overrightarrow{\boldsymbol{\sigma}}, \overrightarrow{\boldsymbol{W}}),
\end{aligned}
$$

and

$$
\begin{aligned}
C^{\dagger}(\overrightarrow{\boldsymbol{W}}) & =\max _{\overrightarrow{\boldsymbol{P}} \in \overrightarrow{\mathcal{P}}(\overrightarrow{\mathcal{X}})} \bar{I}(\overrightarrow{\boldsymbol{P}}, \overrightarrow{\boldsymbol{W}}) \\
& =\max _{\overrightarrow{\boldsymbol{P}} \in \overrightarrow{\mathcal{P}}(\overrightarrow{\mathcal{X}})} \min _{\overrightarrow{\boldsymbol{\sigma}} \in \overrightarrow{\mathcal{S}}(\overrightarrow{\mathcal{H}})} \bar{J}(\overrightarrow{\boldsymbol{P}}, \overrightarrow{\boldsymbol{\sigma}}, \overrightarrow{\boldsymbol{W}}) .
\end{aligned}
$$

Remark 5 The formula obtained by Verdú and Han [5] for a sequence of classical channels $\mathbf{W}=\left\{W^{(n)}\right\}_{n=1}^{\infty}$ is

$$
C(\mathbf{W})=\sup _{\mathbf{X}} \underline{I}(\mathbf{X} ; \mathbf{Y})
$$

where the supremum is taken over all possible input sequences $\mathbf{X}=\left\{X^{(n)}\right\}$, and $\mathbf{Y}=$ $\left\{Y^{(n)}\right\}$ denotes the output sequences corresponding to $\mathbf{X}$. In addition, they showed that the strong converse holds for $\mathbf{W}$ if and only if $\sup _{\mathbf{X}} \underline{I}(\mathbf{X} ; \mathbf{Y})=\sup _{\mathbf{X}} \bar{I}(\mathbf{X} ; \mathbf{Y})$. In the process of proving this, they have essentially shown that

$$
C^{\dagger}(\mathbf{W})=\sup _{\mathbf{X}} \bar{I}(\mathbf{X} ; \mathbf{Y})
$$

even though $C^{\dagger}(\mathbf{W})$ does not explicitly appear in that paper. Note that the supremums in these expressions can be replaced with maximums (see Remark 7 below), and our expressions (71) and (9) are the quantum extensions of (11) and (12).

Remark 6 In the classical case, let

$$
\begin{aligned}
& \bar{J}(\mathbf{X}, \hat{\mathbf{Y}}, \mathbf{W}) \stackrel{\text { def }}{=} \mathrm{p}-\limsup _{n \rightarrow \infty} \frac{1}{n} \log \frac{W^{(n)}\left(Y^{(n)} \mid X^{(n)}\right)}{P_{\hat{Y}^{(n)}}\left(Y^{(n)}\right)}, \\
& \underline{J}(\mathbf{X}, \hat{\mathbf{Y}}, \mathbf{W}) \stackrel{\text { def }}{=} \mathrm{p}-\liminf _{n \rightarrow \infty} \frac{1}{n} \log \frac{W^{(n)}\left(Y^{(n)} \mid X^{(n)}\right)}{P_{\hat{Y}^{(n)}}\left(Y^{(n)}\right)},
\end{aligned}
$$

where $\hat{Y}^{(n)}$ is an arbitrary random variable with a probability distribution $P_{\hat{Y}^{(n)}}$ taking values in a common set with $Y^{(n)}$. Then we have

$$
\begin{aligned}
\bar{J}(\mathbf{X}, \hat{\mathbf{Y}}, \mathbf{W}) & \geq \mathrm{p}-\limsup _{n \rightarrow \infty} \frac{1}{n} \log \frac{W^{(n)}\left(Y^{(n)} \mid X^{(n)}\right)}{P_{Y^{(n)}}\left(Y^{(n)}\right)}+\mathrm{p}-\liminf _{n \rightarrow \infty} \frac{1}{n} \log \frac{P_{Y^{(n)}}\left(Y^{(n)}\right)}{P_{\hat{Y}^{(n)}}\left(Y^{(n)}\right)} \\
& =\bar{I}(\mathbf{X} ; \mathbf{Y})+\underline{D}(\mathbf{Y} \| \hat{\mathbf{Y}}), \\
\underline{J}(\mathbf{X}, \hat{\mathbf{Y}}, \mathbf{W}) & \geq \mathrm{p}-\liminf _{n \rightarrow \infty} \frac{1}{n} \log \frac{W^{(n)}\left(Y^{(n)} \mid X^{(n)}\right)}{P_{Y^{(n)}}\left(Y^{(n)}\right)}+\mathrm{p}-\liminf _{n \rightarrow \infty} \frac{1}{n} \log \frac{P_{Y^{(n)}}\left(Y^{(n)}\right)}{P_{\hat{Y}^{(n)}}\left(Y^{(n)}\right)} \\
& =\underline{I}(\mathbf{X} ; \mathbf{Y})+\underline{D}(\mathbf{Y} \| \hat{\mathbf{Y}}),
\end{aligned}
$$


where $\underline{D}(\mathbf{Y} \| \hat{\mathbf{Y}})$ is the spectral inf-divergence rate $[0]$ between $\mathbf{Y}$ and $\hat{\mathbf{Y}}$. Since $\underline{D}(\mathbf{Y} \| \hat{\mathbf{Y}}) \geq \mathbf{0}$ always holds, we have

$$
\begin{aligned}
& \bar{I}(\mathbf{X} ; \mathbf{Y})=\min _{\hat{\mathbf{Y}}} \bar{J}(\mathbf{X}, \hat{\mathbf{Y}}, \mathbf{W}), \quad \text { and } \\
& \underline{I}(\mathbf{X} ; \mathbf{Y})=\min _{\hat{\mathbf{Y}}} \underline{J}(\mathbf{X}, \hat{\mathbf{Y}}, \mathbf{W}),
\end{aligned}
$$

which yield similar expressions to (81) and (10) from (11) and (12). In the quantum case, on the other hand, it is not clear whether the corresponding equations $\bar{I}(\overrightarrow{\boldsymbol{P}}, \overrightarrow{\boldsymbol{W}})=\min _{\overrightarrow{\boldsymbol{\sigma}}} \bar{J}(\overrightarrow{\boldsymbol{P}}, \overrightarrow{\boldsymbol{\sigma}}, \overrightarrow{\boldsymbol{W}})$ and $\underline{I}(\overrightarrow{\boldsymbol{P}}, \overrightarrow{\boldsymbol{W}})=\min _{\overrightarrow{\boldsymbol{\sigma}}} \underline{J}(\overrightarrow{\boldsymbol{P}}, \overrightarrow{\boldsymbol{\sigma}}, \overrightarrow{\boldsymbol{W}})$ generally hold. Nevertheless the expressions for $C(\overrightarrow{\boldsymbol{W}})$ and $C^{\dagger}(\overrightarrow{\boldsymbol{W}})$ in Theorem 1 always hold.

Remark 7 If a classical or quantum information-spectrum quantity includes a sequence of variables, the supremum (infimum, resp.) (e.g. (11), (12) ) with respect to the variables can always be replaced with the maximum (miminum) due to the following lemma. Thus we do not need to care about the attainability of such a supremum (infimum).

Lemma 1 Suppose that we are given a sequence $\left\{\mathcal{F}_{n}\right\}_{n=1}^{\infty}$, where each $\mathcal{F}_{n}$ is a nonempty set consisting of monotonically nondecreasing functions defined on $\mathbb{R}$, and let $\overrightarrow{\mathcal{F}}$ denote the totality of sequences $\overrightarrow{\boldsymbol{f}}=\left\{f_{n}\right\}_{n=1}^{\infty}$ of functions $f_{n} \in \mathcal{F}_{n}$; in other words, $\overrightarrow{\mathcal{F}}$ is the direct product $\prod_{n=1}^{\infty} \mathcal{F}_{n}$ of $\left\{\mathcal{F}_{n}\right\}$. For each $\overrightarrow{\boldsymbol{f}} \in \overrightarrow{\mathcal{F}}$ and $x \in \mathbb{R}$, let

$$
\begin{aligned}
& {[\overrightarrow{\boldsymbol{f}}]_{x}^{-} \stackrel{\text { def }}{=} \sup \left\{a \mid \limsup _{n \rightarrow \infty} f_{n}(a) \leq x\right\} \in \mathbb{R} \cup\{\infty,-\infty\},} \\
& {[\overrightarrow{\boldsymbol{f}}]_{x}^{+} \stackrel{\text { def }}{=} \inf \left\{a \mid \liminf _{n \rightarrow \infty} f_{n}(a) \geq x\right\} \in \mathbb{R} \cup\{\infty,-\infty\} .}
\end{aligned}
$$

Then the supremums and infimums of

$$
\sup _{\overrightarrow{\boldsymbol{f}}}[\overrightarrow{\boldsymbol{f}}]_{x}^{-}, \quad \sup _{\overrightarrow{\boldsymbol{f}}}[\overrightarrow{\boldsymbol{f}}]_{x}^{+}, \quad \inf _{\overrightarrow{\boldsymbol{f}}}[\overrightarrow{\boldsymbol{f}}]_{x}^{-} \quad \text { and } \quad \inf _{\overrightarrow{\boldsymbol{f}}}[\overrightarrow{\boldsymbol{f}}]_{x}^{+}
$$

are always attainable in $\overrightarrow{\mathcal{F}}$.

Proof: See Appendix I.

In the situation of Thorem 1] for instance, the lemma is applied to sequences of functions $\overrightarrow{\boldsymbol{f}}=\left\{f_{n}\right\}_{n=1}^{\infty}$ of the form

$$
f_{n}(a)=\sum_{x^{n} \in \mathcal{X}^{(n)}} P^{(n)}\left(x^{n}\right) \operatorname{Tr}\left[W_{x^{n}}^{(n)}\left\{W_{x^{n}}^{(n)}-e^{n a} \sigma^{(n)} \leq 0\right\}\right],
$$

for which we have $[\overrightarrow{\boldsymbol{f}}]_{0}^{-}=\underline{J}(\overrightarrow{\boldsymbol{P}}, \overrightarrow{\boldsymbol{\sigma}}, \overrightarrow{\boldsymbol{W}})$ and $[\overrightarrow{\boldsymbol{f}}]_{1}^{+}=\bar{J}(\overrightarrow{\boldsymbol{P}}, \overrightarrow{\boldsymbol{\sigma}}, \overrightarrow{\boldsymbol{W}})$. Note that the monotonicity of these functions follows from an argument in section 3 of [10]. 


\section{$4 \quad$ Lemmas for proving Theorem 1}

We need three lemmas. The first one is the key operator inequality to prove the second, while the second and third lemmas are directly used to prove the theorem. Throughout this paper the generalized inverse of a nonnegative operator $A$ is simply denoted by $A^{-1}$; i.e., $A^{-1}$ is the nonnegative operator such that $A A^{-1}=A^{-1} A=$ $P_{A}=P_{A^{-1}}$ where $P_{A}$ and $P_{A^{-1}}$ denote the orthogonal projections onto the ranges of $A$ and $A^{-1}$.

Lemma 2 For any positive number $c$ and any operators $0 \leq S \leq I$ and $T \geq 0$, we have

$$
I-\sqrt{S+T}^{-1} S \sqrt{S+T}^{-1} \leq(1+c)(I-S)+\left(2+c+c^{-1}\right) T .
$$

Proof: $\quad$ Let $P$ be the orthogonal projection onto the range of $S+T$. Then $P$ commutes both $S$ and $T$, and hence it is enough to prove

$$
\begin{aligned}
& P\left[I-\sqrt{S+T}^{-1} S \sqrt{S+T}^{-1}\right] P \leq P\left[(1+c)(I-S)+\left(2+c+c^{-1}\right) T\right] P, \quad \text { and } \\
& P^{\perp}\left[I-\sqrt{S+T}^{-1} S \sqrt{S+T}^{-1}\right] P^{\perp} \leq P^{\perp}\left[(1+c)(I-S)+\left(2+c+c^{-1}\right) T\right] P^{\perp},
\end{aligned}
$$

where $P^{\perp}=I-P$. Since $P^{\perp} S=P^{\perp} T=P^{\perp} \sqrt{S+T}^{-1}=0$, the second inequality is trivial. Thus, we have only to show the first one or, equivalently, to show (15) in the case when the range of $S+T$ is $\mathcal{H}$. Substituting $A=\sqrt{T}$ and $B=\sqrt{T}\left(\sqrt{S+T}^{-1}-I\right)$ into the general operator inequality $A^{*} B+B^{*} A \leq c^{-1} A^{*} A+c B^{*} B$, which follows from $(A-c B)^{*}(A-c B) \geq 0$, we have

$$
\begin{aligned}
& T\left(\sqrt{S+T}^{-1}-I\right)+\left(\sqrt{S+T}^{-1}-I\right) T \\
\leq & c^{-1} T+c\left(\sqrt{S+T}^{-1}-I\right) T\left(\sqrt{S+T}^{-1}-I\right) .
\end{aligned}
$$

In addition, since the function $f(x)=\sqrt{x}$ is an operator monotone function and $0 \leq S \leq I$, we have

$$
\sqrt{S+T} \geq \sqrt{S} \geq S .
$$

Now the desired inequality is proved as follows:

$$
\begin{aligned}
& I-\sqrt{S+T}^{-1} S \sqrt{S+T}^{-1}=\sqrt{S+T}^{-1} T \sqrt{S+T}^{-1} \\
= & T+T\left(\sqrt{S+T}^{-1}-I\right)+\left(\sqrt{S+T}^{-1}-I\right) T+\left(\sqrt{S+T}^{-1}-I\right) T\left(\sqrt{S+T}^{-1}-I\right) \\
\leq & \left(1+c^{-1}\right) T+(1+c)\left(\sqrt{S+T}^{-1}-I\right) T\left(\sqrt{S+T}^{-1}-I\right) \\
\leq & \left(1+c^{-1}\right) T+(1+c)\left(\sqrt{S+T}^{-1}-I\right)(S+T)\left(\sqrt{S+T}^{-1}-I\right) \\
= & \left(1+c^{-1}\right) T+(1+c)(I+S+T-2 \sqrt{S+T}) \\
\leq & \left(1+c^{-1}\right) T+(1+c)(I+S+T-2 S) \\
= & (1+c)(I-S)+\left(2+c+c^{-1}\right) T,
\end{aligned}
$$


where the first inequality follows from (16) and the third inequality follows from (17).

Lemma 3 For any $n \in \mathbb{N}, a \in \mathbb{R}, N \in \mathbb{N}, P^{(n)} \in \mathcal{P}\left(\mathcal{X}^{(n)}\right)$ and $c>0$, there exists a code $\Phi^{(n)} \in \mathfrak{C}\left(W^{(n)}\right)$ such that $\left|\Phi^{(n)}\right|=N$ and

$$
\begin{aligned}
\mathrm{P}_{\mathrm{e}}\left[\Phi^{(n)}\right] \leq & (1+c) \sum_{x^{n} \in \mathcal{X}^{(n)}} P^{(n)}\left(x^{n}\right) \operatorname{Tr}\left[W_{x^{n}}^{(n)}\left\{W_{x^{n}}^{(n)}-e^{n a} W_{P^{(n)}}^{(n)} \leq 0\right\}\right] \\
& +\left(2+c+c^{-1}\right) e^{-n a} N,
\end{aligned}
$$

where $W_{P^{(n)}}^{(n)}$ is defined by (6)).

Proof: We prove the lemma by a random coding method. Given $n, a, N, P^{(n)}$ and an encoder $\varphi^{(n)}:\{1, \ldots, N\} \rightarrow \mathcal{X}^{(n)}$, define the decoding POVM $Y^{(n)}=\left\{Y_{i}^{(n)}\right\}_{i=1}^{N}$ by

$$
Y_{i}^{(n)} \stackrel{\text { def }}{=}\left(\sum_{j=1}^{N} \pi_{j}\right)^{-\frac{1}{2}} \pi_{i}\left(\sum_{j=1}^{N} \pi_{j}\right)^{-\frac{1}{2}}
$$

where

$$
\pi_{i} \stackrel{\text { def }}{=}\left\{W_{\varphi^{(n)}(i)}^{(n)}-e^{n a} W_{P^{(n)}}^{(n)}>0\right\} .
$$

Denoting the average error probability $\mathrm{P}_{\mathrm{e}}\left[\Phi^{(n)}\right]$ of the code $\Phi^{(n)}=\left(N, \varphi^{(n)}, Y^{(n)}\right)$ by $\mathrm{P}_{\mathrm{e}}\left[\varphi^{(n)}\right]$, we have

$$
\begin{aligned}
\mathrm{P}_{\mathrm{e}}\left[\varphi^{(n)}\right] & =\frac{1}{N} \sum_{i=1}^{N} \operatorname{Tr}\left[W_{\varphi^{(n)}(i)}^{(n)}\left(I-Y_{i}^{(n)}\right)\right] \\
& \leq \frac{1}{N} \sum_{i=1}^{N} \operatorname{Tr}\left[W_{\varphi^{(n)}(i)}^{(n)}\left((1+c)\left(I-\pi_{i}\right)+\left(2+c+c^{-1}\right) \sum_{j \neq i} \pi_{j}\right)\right],
\end{aligned}
$$

which follows from Lemma 2, Now suppose that an encoder $\varphi^{(n)}$ is randomly generated according to the probability distribution $P_{\mathrm{rc}}^{(n)}\left(\varphi^{(n)}\right)=P^{(n)}\left(\varphi^{(n)}(1)\right) \cdots P^{(n)}\left(\varphi^{(n)}(N)\right)$. The expectation of $\mathrm{P}_{\mathrm{e}}\left[\varphi^{(n)}\right]$ under $P_{\mathrm{rc}}^{(n)}$ is then bounded from above as

$$
\begin{aligned}
E_{\mathrm{rc}}^{(n)} \mathrm{P}_{\mathrm{e}}\left[\varphi^{(n)}\right] \leq & E_{\mathrm{rc}}^{(n)} \frac{1+c}{N} \sum_{i=1}^{N} \operatorname{Tr}\left[W_{\varphi^{(n)}(i)}^{(n)}\left\{W_{\varphi^{(n)}(i)}^{(n)}-e^{n a} W_{P^{(n)}}^{(n)} \leq 0\right\}\right] \\
& +E_{\mathrm{rc}}^{(n)} \frac{2+c+c^{-1}}{N} \sum_{i=1}^{N} \sum_{j \neq i} \operatorname{Tr}\left[W_{\varphi^{(n)}(i)}^{(n)}\left\{W_{\varphi^{(n)}(j)}^{(n)}-e^{n a} W_{P^{(n)}}^{(n)}>0\right\}\right] \\
= & (1+c) \sum_{x^{n}} P^{(n)}\left(x^{n}\right) \operatorname{Tr}\left[W_{x^{n}}^{(n)}\left\{W_{x^{n}}^{(n)}-e^{n a} W_{P^{(n)}}^{(n)} \leq 0\right\}\right] \\
& +\left(2+c+c^{-1}\right) N \sum_{x^{n}} P^{(n)}\left(x^{n}\right) \operatorname{Tr}\left[W_{P(n)}^{(n)}\left\{W_{x^{n}}^{(n)}-e^{n a} W_{P^{(n)}}^{(n)}>0\right\}\right] .
\end{aligned}
$$


Substituting $A=W_{x^{n}}^{(n)}-e^{n a} W_{P^{(n)}}^{(n)}$ into $\operatorname{Tr}[A\{A>0\}] \geq 0$, the second term of (22) is further evaluated by

$$
\begin{aligned}
& \sum_{x^{n}} P^{(n)}\left(x^{n}\right) \operatorname{Tr}\left[W_{P^{(n)}}^{(n)}\left\{W_{x^{n}}^{(n)}-e^{n a} W_{P^{(n)}}^{(n)}>0\right\}\right] \\
\leq & e^{-n a} \sum_{x^{n}} P^{(n)}\left(x^{n}\right) \operatorname{Tr}\left[W_{x^{n}}^{(n)}\left\{W_{x^{n}}^{(n)}-e^{n a} W_{P^{(n)}}^{(n)}>0\right\}\right] \\
\leq & e^{-n a} .
\end{aligned}
$$

Thus the existence of $\varphi^{(n)}$ for which the code $\Phi^{(n)}=\left(N, \varphi^{(n)}, Y^{(n)}\right)$ satisfies (18) has been proved.

Remark 8 In deriving the direct part of the general capacity formula for classical channels, Verdú and Han [5] invoked the so-called Feinstein's lemma (Theorem 1 in [5]; see the next remark) which ensures the existence of a code satisfying

$$
\mathrm{P}_{\mathrm{e}}\left[\Phi^{(n)}\right] \leq \operatorname{Prob}\left\{\frac{1}{n} \log \frac{W^{(n)}\left(Y^{(n)} \mid X^{(n)}\right)}{P_{Y^{(n)}}\left(Y^{(n)}\right)} \leq a\right\}+e^{-n a} N .
$$

Lemma 3 above can be regarded as a quantum analogue of Feinstein's lemma, although the coefficients there are a bit larger.

Remark 9 Historically, it seems that Shannon [19] was the first to explicitly formulate the inequality (23). He used a random coding argument to prove that there exists a code whose average error probability satisfies (23). On the other hand, Blackwell et al. 20] showed that the same inequality is also satisfiable for the maximum error probability. They proved this by refining Feinstein's non-random packing argument, which is well known to have been used in the first rigorous proof of the coding theorem for discrete memoryless channels [21]. This course of things makes some people to call the theorem concerning (23) "Feinstein's lemma", sometimes only for the maximum error probability and sometimes for both criteria (cf. [5]). We note that the original proof of Feinstein does not yield the general capacity formula, and the refinement mede by Blackwell et al. is essential in this respect. Our Lemma 3 corresponds to Shannon's one, while an attempt toward a quantum extension of the result of Blackwell et al. has been made in [13, 14]. The result obtained there is unfortunately not general enough to prove the direct part of the general formula (7), but is of a particular interest itself; see Remark 14 below.

Remark 10 Letting $A \stackrel{\text { def }}{=} \sum_{x^{n} \in \mathcal{X}^{(n)}} P^{(n)}\left(x^{n}\right) \operatorname{Tr}\left[W_{x^{n}}^{(n)}\left\{W_{x^{n}}^{(n)}-e^{n a} W_{P^{(n)}}^{(n)} \leq 0\right\}\right]$ and $B \stackrel{\text { def }}{=} e^{-n a} N$, the RHS of (18) is minimized at $c=\sqrt{\frac{B}{A+B}}$, which proves the existence of a code satisfying

$$
\mathrm{P}_{\mathrm{e}}\left[\Phi^{(n)}\right] \leq A+2 B+2 \sqrt{B(A+B)}
$$


Lemma 4 For any $n \in \mathbb{N}$ and any code $\Phi^{(n)} \in \mathfrak{C}\left(W^{(n)}\right)$ with $\left|\Phi^{(n)}\right|=N$, there exists a probability distribution $P^{(n)} \in \mathcal{P}\left(\mathcal{X}^{(n)}\right)$ such that for any $a \in \mathbb{R}$ and $\sigma^{(n)} \in \mathcal{S}\left(\mathcal{H}^{(n)}\right)$

$$
\mathrm{P}_{\mathrm{e}}\left[\Phi^{(n)}\right] \geq \sum_{x^{n} \in \mathcal{X}^{(n)}} P^{(n)}\left(x^{n}\right) \operatorname{Tr}\left[W_{x^{n}}^{(n)}\left\{W_{x^{n}}^{(n)}-e^{n a} \sigma^{(n)} \leq 0\right\}\right]-\frac{e^{n a}}{N} .
$$

Proof: Remember that for any operators $A \geq 0$ and $0 \leq T \leq I$,

$$
\operatorname{Tr}[A T] \leq \operatorname{Tr}[A\{A>0\}],
$$

which is the essence of the quantum Neyman-Pearson lemma [8, 9, 10]. Then we see that for any code $\Phi^{(n)}=\left(N, \varphi^{(n)}, Y^{(n)}\right)$,

$$
\begin{aligned}
\operatorname{Tr}\left[\left(W_{\varphi^{(n)}(i)}^{(n)}-e^{n a} \sigma^{(n)}\right) Y_{i}^{(n)}\right] & \leq \operatorname{Tr}\left[\left(W_{\varphi^{(n)}(i)}^{(n)}-e^{n a} \sigma^{(n)}\right)\left\{W_{\varphi^{(n)}(i)}^{(n)}-e^{n a} \sigma^{(n)}>0\right\}\right] \\
& \leq \operatorname{Tr}\left[W_{\varphi^{(n)}(i)}^{(n)}\left\{W_{\varphi^{(n)}(i)}^{(n)}-e^{n a} \sigma^{(n)}>0\right\}\right] .
\end{aligned}
$$

This is rewritten as

$\operatorname{Tr}\left[W_{\varphi^{(n)}(i)}^{(n)}\left(I-Y_{i}^{(n)}\right)\right] \geq \operatorname{Tr}\left[W_{\varphi^{(n)}(i)}^{(n)}\left\{W_{\varphi^{(n)}(i)}^{(n)}-e^{n a} \sigma^{(n)} \leq 0\right\}\right]-e^{n a} \operatorname{Tr}\left[\sigma^{(n)} Y_{i}^{(n)}\right]$,

and hence we have

$$
\begin{aligned}
\mathrm{P}_{\mathrm{e}}\left[\Phi^{(n)}\right] & \geq \frac{1}{N} \sum_{i=1}^{N} \operatorname{Tr}\left[W_{\varphi^{(n)}(i)}^{(n)}\left\{W_{\varphi^{(n)}(i)}^{(n)}-e^{n a} \sigma^{(n)} \leq 0\right\}\right]-\frac{e^{n a}}{N} \sum_{i=1}^{N} \operatorname{Tr}\left[\sigma^{(n)} Y_{i}^{(n)}\right] \\
& \geq \frac{1}{N} \sum_{i=1}^{N} \operatorname{Tr}\left[W_{\varphi^{(n)}(i)}^{(n)}\left\{W_{\varphi^{(n)}(i)}^{(n)}-e^{n a} \sigma^{(n)} \leq 0\right\}\right]-\frac{e^{n a}}{N} .
\end{aligned}
$$

We thus have (24) by letting $P^{(n)}$ be the empirical distribution for the $N$ points $\left(\varphi^{(n)}(1), \ldots, \varphi^{(n)}(N)\right)$.

Remark 11 Lemma 4 in the case of $\sigma^{(n)}=W_{P(n)}^{(n)}$ is just the quantum analogue of Theorem 4 in [5] which evaluates the error probability of a code as

$$
\mathrm{P}_{\mathrm{e}}\left[\Phi^{(n)}\right] \geq \operatorname{Prob}\left\{\frac{1}{n} \log \frac{W^{(n)}\left(Y^{(n)} \mid X^{(n)}\right)}{P_{Y^{(n)}}\left(Y^{(n)}\right)} \leq a\right\}-\frac{e^{n a}}{N} .
$$

Our results might seem to be still incomplete in comparison with the beautiful duality between (23) and (26). 


\section{$5 \quad$ Proof of Theorem 1}

Now Theorem 1 is proved as follows. We first show the inequality

$$
C(\overrightarrow{\boldsymbol{W}}) \geq \max _{\overrightarrow{\boldsymbol{P}} \in \overrightarrow{\mathcal{P}}(\overrightarrow{\mathcal{X}})} I(\overrightarrow{\boldsymbol{P}}, \overrightarrow{\boldsymbol{W}}) .
$$

Here we can assume that the RHS is strictly positive since otherwise the inequality is trivial. Suppose that we are given a sequence $\overrightarrow{\boldsymbol{P}}=\left\{P^{(n)}\right\} \in \overrightarrow{\mathcal{P}}(\overrightarrow{\mathcal{X}})$ and a number $R$ such that $0<R<\underline{I}(\overrightarrow{\boldsymbol{P}}, \overrightarrow{\boldsymbol{W}})$. Setting $N=\left\lceil e^{n R}\right\rceil$ in Lemma 3, it follows that for each real number $a$ and $c>0$ there exists a sequence of codes $\overrightarrow{\boldsymbol{\Phi}}=\left\{\Phi^{(n)}\right\} \in \overrightarrow{\mathfrak{C}}(\overrightarrow{\boldsymbol{W}})$ such that $\left|\Phi^{(n)}\right|=\left\lceil e^{n R}\right\rceil$ and

$$
\begin{aligned}
\mathrm{P}_{\mathrm{e}}\left[\Phi^{(n)}\right] \leq & (1+c) \sum_{x^{n} \in \mathcal{X}^{(n)}} P^{(n)}\left(x^{n}\right) \operatorname{Tr}\left[W_{x^{n}}^{(n)}\left\{W_{x^{n}}^{(n)}-e^{n a} W_{P^{(n)}}^{(n)} \leq 0\right\}\right] \\
& +\left(2+c+c^{-1}\right) e^{-n a}\left\lceil e^{n R}\right\rceil
\end{aligned}
$$

for every $n$. Recalling the definition of $\underline{I}(\overrightarrow{\boldsymbol{P}}, \overrightarrow{\boldsymbol{W}})$, we see that the first term of the RHS goes to 0 as $n \rightarrow \infty$ for any $a<\underline{I}(\overrightarrow{\boldsymbol{P}}, \overrightarrow{\boldsymbol{W}})$, while the second term goes to 0 for any $a>R$. Hence, letting $a$ lie in $R<a<\underline{I}(\overrightarrow{\boldsymbol{P}}, \overrightarrow{\boldsymbol{W}})$, the existence of a $\overrightarrow{\boldsymbol{\Phi}}$ satisfying $\liminf _{n \rightarrow \infty} \frac{1}{N} \log \Phi^{(n)} \mid \geq R$ and $\lim _{n \rightarrow \infty} \mathrm{P}_{\mathrm{e}}\left[\Phi^{(n)}\right]=0$ is shown. This implies that $R \leq C(\overrightarrow{\boldsymbol{W}})$ for any $0<R<\underline{I}(\overrightarrow{\boldsymbol{P}}, \overrightarrow{\boldsymbol{W}})$, and completes the proof of (27).

Next we prove

$$
C^{\dagger}(\overrightarrow{\boldsymbol{W}}) \geq \max _{\overrightarrow{\boldsymbol{P}} \in \overrightarrow{\mathcal{P}}(\overrightarrow{\mathcal{X}})} \bar{I}(\overrightarrow{\boldsymbol{P}}, \overrightarrow{\boldsymbol{W}})
$$

We can assume that $C^{\dagger}(\overrightarrow{\boldsymbol{W}})<\infty$ since otherwise the inequality is trivial. Let $R$ be an arbitrary number greater than $C^{\dagger}(\overrightarrow{\boldsymbol{W}})$. Then for each $a$ and $c>0$ there exists a sequence of codes $\overrightarrow{\boldsymbol{\Phi}}=\left\{\Phi^{(n)}\right\} \in \overrightarrow{\mathfrak{C}}(\overrightarrow{\boldsymbol{W}})$ such that $\left|\Phi^{(n)}\right|=\left\lceil e^{n R}\right\rceil$ and (28) holds for every $n$. From $\lim _{n \rightarrow \infty} \frac{1}{n} \log \left|\Phi^{(n)}\right|=R>C^{\dagger}(\overrightarrow{\boldsymbol{W}}), \mathrm{P}_{\mathrm{e}}\left[\Phi^{(n)}\right]$ must go to 1 as $n \rightarrow \infty$, and therefore (28) yields that for any $a>R$

$$
1 \leq(1+c) \liminf _{n \rightarrow \infty} \sum_{x^{n} \in \mathcal{X}^{(n)}} P^{(n)}\left(x^{n}\right) \operatorname{Tr}\left[W_{x^{n}}^{(n)}\left\{W_{x^{n}}^{(n)}-e^{n a} W_{P^{(n)}}^{(n)} \leq 0\right\}\right] .
$$

Since $c>0$ is arbitrary, $\sum_{x^{n} \in \mathcal{X}^{(n)}} P^{(n)}\left(x^{n}\right) \operatorname{Tr}\left[W_{x^{n}}^{(n)}\left\{W_{x^{n}}^{(n)}-e^{n a} W_{P^{(n)}}^{(n)} \leq 0\right\}\right]$ converges to 1 and hence $a \geq \bar{I}(\overrightarrow{\boldsymbol{P}}, \overrightarrow{\boldsymbol{W}})$. We thus have $a \geq \bar{I}(\overrightarrow{\boldsymbol{P}}, \overrightarrow{\boldsymbol{W}})$ for $\forall a>\forall R>$ $C^{\dagger}(\overrightarrow{\boldsymbol{W}})$, and (29) has been proved.

Let us proceed to prove the converse inequality

$$
C(\overrightarrow{\boldsymbol{W}}) \leq \max _{\overrightarrow{\boldsymbol{P}} \in \overrightarrow{\mathcal{P}}(\overrightarrow{\mathcal{X}})} \min _{\overrightarrow{\boldsymbol{\sigma}} \in \overrightarrow{\mathcal{S}}(\overrightarrow{\mathcal{H}})} \underline{J}(\overrightarrow{\boldsymbol{P}}, \overrightarrow{\boldsymbol{\sigma}}, \overrightarrow{\boldsymbol{W}}) .
$$

Let $R<C(\overrightarrow{\boldsymbol{W}})$. Then there exists a sequence of codes $\overrightarrow{\boldsymbol{\Phi}}=\left\{\Phi^{(n)}\right\} \in \overrightarrow{\mathfrak{C}}(\overrightarrow{\boldsymbol{W}})$ satisfying

$$
\liminf _{n \rightarrow \infty} \frac{1}{n} \log \left|\Phi^{(n)}\right|>R \text { and } \quad \lim _{n \rightarrow \infty} \mathrm{P}_{\mathrm{e}}\left[\Phi^{(n)}\right]=0 .
$$


From Lemma 4, there exists a $\overrightarrow{\boldsymbol{P}}=\left\{P^{(n)}\right\}$ such that for any $n \in \mathbb{N}$ and $\overrightarrow{\boldsymbol{\sigma}}=\left\{\sigma^{(n)}\right\}$,

$$
\begin{aligned}
\sum_{x^{n} \in \mathcal{X}^{(n)}} P^{(n)}\left(x^{n}\right) \operatorname{Tr}\left[W_{x^{n}}^{(n)}\left\{W_{x^{n}}^{(n)}-e^{n R} \sigma^{(n)} \leq 0\right\}\right] & \leq \mathrm{P}_{\mathrm{e}}\left[\Phi^{(n)}\right]+\frac{e^{n R}}{\left|\Phi^{(n)}\right|} \\
& \rightarrow 0 \text { as } n \rightarrow \infty
\end{aligned}
$$

This implies that $R \leq \min _{\overrightarrow{\boldsymbol{\sigma}}} \underline{J}(\overrightarrow{\boldsymbol{P}}, \overrightarrow{\boldsymbol{\sigma}}, \overrightarrow{\boldsymbol{W}})$ for some $\overrightarrow{\boldsymbol{P}}$. Therefore, we have

$$
R \leq \max _{\overrightarrow{\boldsymbol{P}} \in \overrightarrow{\mathcal{P}}(\overrightarrow{\boldsymbol{W}})} \min _{\overrightarrow{\boldsymbol{\sigma}}} \underline{J}(\overrightarrow{\boldsymbol{P}}, \overrightarrow{\boldsymbol{\sigma}}, \overrightarrow{\boldsymbol{W}})
$$

for any $R<C(\overrightarrow{\boldsymbol{W}})$, and (30) has been proved. Similarly, we can prove

$$
C^{\dagger}(\overrightarrow{\boldsymbol{W}}) \leq \max _{\overrightarrow{\boldsymbol{P}} \in \overrightarrow{\mathcal{P}}(\overrightarrow{\mathcal{X}})} \min _{\overrightarrow{\boldsymbol{\sigma}} \in \overrightarrow{\mathcal{S}}(\overrightarrow{\mathcal{H}})} \bar{J}(\overrightarrow{\boldsymbol{P}}, \overrightarrow{\boldsymbol{\sigma}}, \overrightarrow{\boldsymbol{W}})
$$

The remaining parts

$$
\max _{\overrightarrow{\boldsymbol{P}} \in \overrightarrow{\mathcal{P}}(\overrightarrow{\mathcal{X}})} I(\overrightarrow{\boldsymbol{P}}, \overrightarrow{\boldsymbol{W}}) \geq \max _{\overrightarrow{\boldsymbol{P}} \in \overrightarrow{\mathcal{P}}(\overrightarrow{\mathcal{X}})} \min _{\overrightarrow{\boldsymbol{\sigma}} \in \overrightarrow{\mathcal{S}}(\overrightarrow{\mathcal{H}})} \underline{J}(\overrightarrow{\boldsymbol{P}}, \overrightarrow{\boldsymbol{\sigma}}, \overrightarrow{\boldsymbol{W}})
$$

and

$$
\max _{\overrightarrow{\boldsymbol{P}} \in \overrightarrow{\mathcal{P}}(\overrightarrow{\mathcal{X}})} \bar{I}(\overrightarrow{\boldsymbol{P}}, \overrightarrow{\boldsymbol{W}}) \geq \max _{\overrightarrow{\boldsymbol{P}} \in \overrightarrow{\mathcal{P}}(\overrightarrow{\mathcal{X}})} \min _{\overrightarrow{\boldsymbol{\sigma}} \in \overrightarrow{\mathcal{S}}(\overrightarrow{\mathcal{H}})} \bar{J}(\overrightarrow{\boldsymbol{P}}, \overrightarrow{\boldsymbol{\sigma}}, \overrightarrow{\boldsymbol{W}})
$$

are obvious from the definitions.

\section{Stationary memoryless case}

In this section we demonstrate how the general formulas given in Theorem 1 leads to the following coding theorem for stationary memoryless channels.

Theorem 2 Let $W: \mathcal{X} \rightarrow \mathcal{S}(\mathcal{H})$ be an arbitrary channel and consider its stationary memoryless extension:

$$
\begin{aligned}
& \mathcal{H}^{(n)}=\mathcal{H}^{\otimes n}, \quad \mathcal{X}^{(n)}=\mathcal{X}^{n}, \quad \text { and } \\
& W_{x^{n}}^{(n)}=W_{x_{1}} \otimes \cdots \otimes W_{x_{n}} \quad \text { for } \quad x^{n}=\left(x_{1}, \ldots, x_{n}\right) .
\end{aligned}
$$

Then the capacity of $\overrightarrow{\boldsymbol{W}}=\left\{W^{(n)}\right\}$ is given by

$$
C(\overrightarrow{\boldsymbol{W}})=\sup _{P \in \mathcal{P}(\mathcal{X})} I(P, W)
$$

where

$$
I(P, W) \stackrel{\text { def }}{=} \sum_{x \in \mathcal{X}} P(x) D\left(W_{x} \| W_{P}\right)
$$

with $D(\rho \| \sigma) \stackrel{\text { def }}{=} \operatorname{Tr}[\rho(\log \rho-\log \sigma)]$ being the quantum relative entropy. Furthermore, if $\operatorname{dim} \mathcal{H}<\infty$, then the strong converse holds: $C^{\dagger}(\overrightarrow{\boldsymbol{W}})=C(\overrightarrow{\boldsymbol{W}})$. 
Remark 12 The proof of the strong converse given below relies essentially on the compactness of the closure of the range $\Delta=\left\{W_{x} \mid x \in \mathcal{X}\right\}$, which follows from the finiteness of $\operatorname{dim} \mathcal{H}$. The argument is immediately extended to a certain class of channels with $\operatorname{dim} \mathcal{H}=\infty$ including the case when $\mathcal{X}$ is a finite set, whereas the general condition for the strong converse in the infinite-dimensional case is yet to be studied.

Remark 13 Let $\Gamma$ be a trace-preserving CP map from the trace-class operators on $\mathcal{H}_{1}$ to those on $\mathcal{H}_{2}$. When considering $\Gamma$ as a classical-quantum channel $W: \mathcal{X} \rightarrow$ $\mathcal{S}\left(\mathcal{H}_{2}\right)$ with $\mathcal{X}=\mathcal{S}\left(\mathcal{H}_{1}\right)$, its stationary memoryless extension $W^{(n)}$ is a channel which maps an $n$-tuple $\left(\sigma_{1}, \ldots, \sigma_{n}\right) \in \mathcal{X}^{(n)}=\mathcal{X}^{n}$ of states $\left\{\sigma_{i}\right\} \subset \mathcal{S}\left(\mathcal{H}_{1}\right)$ to the product state $\Gamma\left(\sigma_{1}\right) \otimes \cdots \otimes \Gamma\left(\sigma_{n}\right)$, and the capacity of $\overrightarrow{\boldsymbol{W}}=\left\{W^{(n)}\right\}$ is given by (33). On the other hand, $\Gamma$ has the stationary memoryless extension $\Gamma^{\otimes n}$ as a "quantum-quantum" channel, which defines another classical-quantum channel $\tilde{W}^{(n)}: \tilde{\mathcal{X}}^{(n)} \rightarrow \mathcal{S}\left(\mathcal{H}_{2}{ }^{\otimes n}\right)$ with $\tilde{\mathcal{X}}^{(n)}=\mathcal{S}\left(\mathcal{H}_{1}{ }^{\otimes n}\right)$. Note that $W^{(n)}$ can be regarded as the restriction $\left.\tilde{W}^{(n)}\right|_{\mathcal{X}^{(n)}}$ of $\tilde{W}^{(n)}$ by identifying $\left(\sigma_{1}, \ldots, \sigma_{n}\right) \in \mathcal{X}^{n}$ with $\sigma_{1} \otimes \cdots \otimes \sigma_{n} \in \tilde{\mathcal{X}}^{(n)}$. The capacity $C(\overrightarrow{\tilde{\boldsymbol{W}}})$ of $\overrightarrow{\overrightarrow{\boldsymbol{W}}}=\left\{\tilde{W}^{(n)}\right\}$ is beyond the scope of the preceding theorem, whereas recently the conjecture $C(\overrightarrow{\tilde{\boldsymbol{W}}})=C(\overrightarrow{\boldsymbol{W}})$ together with the more fundamental additivity conjecture has been calling wide attention. See, for instance, [22, 23, 24, 25] and the references cited there.

Historically, the converse part $C(\overrightarrow{\boldsymbol{W}}) \leq \sup _{P \in \mathcal{P}(\mathcal{X})} I(P, W)$ was first established by Holevo's early work [3, 4] which is now often referred to as the Holevo bound, while the direct part $C(\overrightarrow{\boldsymbol{W}}) \geq \sup _{P \in \mathcal{P}(\mathcal{X})} I(P, W)$ was proved much more recently by Holevo [1] and Schumacher-Westmoreland [2]. It should be noted that their proof is based on the representation of $I(P, W)$ as the entropy difference:

$$
I(P, W)=H\left(W_{P}\right)-\sum_{x} P(x) H\left(W_{x}\right)
$$

where $H(\rho) \stackrel{\text { def }}{=}-\operatorname{Tr}[\rho \log \rho]$ is the von Neumann entropy, and hence needs (when $\operatorname{dim} \mathcal{H}=\infty)$ the assumption

$$
H\left(W_{x}\right)<\infty, \quad \forall x \in \mathcal{X} .
$$

See the next section for more details. Our proof given below has the advantage of not needing this finiteness assumption (cf. Remark [16). Note also that in the case when $\operatorname{dim} \mathcal{H}<\infty$ the range of supremum in (33) can be restricted to those $P \in \mathcal{P}(\mathcal{X})$ with $|\operatorname{supp}(P)| \leq \operatorname{dim} \Delta+1$, where $|\operatorname{supp}(P)|$ denotes the number of elements of the support of $P$ and $\Delta \stackrel{\text { def }}{=}\left\{W_{x} \mid x \in \mathcal{X}\right\}$, and that the supremum can be replaced with maximum when $\Delta$ is closed (and hence compact); see [18, 26]. The strong converse $C^{\dagger}(\overrightarrow{\boldsymbol{W}}) \leq \sup _{P \in \mathcal{P}(\mathcal{X})} I(P, W)$ for a finite $\mathcal{X}$ was shown in [15, 7].

Let us begin with considering the (weak) converse

$$
C(\overrightarrow{\boldsymbol{W}}) \leq \sup _{P \in \mathcal{P}(\mathcal{X})} I(P, W)
$$


Lemma 5 For any sequence of channels $\overrightarrow{\boldsymbol{W}}=\left\{W^{(n)}\right\}$ and any sequence of distributions $\overrightarrow{\boldsymbol{P}}=\left\{P^{(n)}\right\}$ we have

$$
\underline{I}(\overrightarrow{\boldsymbol{P}}, \overrightarrow{\boldsymbol{W}}) \leq \liminf _{n \rightarrow \infty} \frac{1}{n} I\left(P^{(n)}, W^{(n)}\right) .
$$

Proof: Given $n, x^{n} \in \mathcal{X}^{(n)}$ and $a \in \mathbb{R}$ arbitrarily, let

$$
\begin{aligned}
& \alpha_{n} \stackrel{\text { def }}{=} \operatorname{Tr}\left[W_{x^{n}}^{(n)}\left\{W_{x^{n}}^{(n)}-e^{n a} W_{P^{(n)}}^{(n)}>0\right\}\right], \\
& \beta_{n} \stackrel{\text { def }}{=} \operatorname{Tr}\left[W_{P^{(n)}}^{(n)}\left\{W_{x^{n}}^{(n)}-e^{n a} W_{P^{(n)}}^{(n)}>0\right\}\right] .
\end{aligned}
$$

Then the monotonicity of the quantum relative entropy yields

$$
\begin{aligned}
D\left(W_{x^{n}}^{(n)} \| W_{P^{(n)}}^{(n)}\right) & \geq \alpha_{n} \log \frac{\alpha_{n}}{\beta_{n}}+\left(1-\alpha_{n}\right) \log \frac{1-\alpha_{n}}{1-\beta_{n}} \\
& \geq-\log 2-\alpha_{n} \log \beta_{n} .
\end{aligned}
$$

On the other hand, we have

$$
0 \leq \operatorname{Tr}\left[\left(W_{x^{n}}^{(n)}-e^{n a} W_{P^{(n)}}^{(n)}\right)\left\{W_{x^{n}}^{(n)}-e^{n a} W_{P^{(n)}}^{(n)}>0\right\}\right]=\alpha_{n}-e^{n a} \beta_{n}
$$

and hence $\beta_{n} \leq e^{-n a} \alpha_{n} \leq e^{-n a}$. We thus obtain $\frac{1}{n} D\left(W_{x^{n}}^{(n)} \| W_{P^{(n)}}^{(n)}\right) \geq-\frac{1}{n} \log 2+a \alpha_{n}$, and taking the expectation w.r.t. $P^{(n)}$ we have

$$
\frac{1}{n} I\left(P^{(n)}, W^{(n)}\right) \geq-\frac{1}{n} \log 2+a \sum_{x^{n}} P^{(n)}\left(x^{n}\right) \operatorname{Tr}\left[W_{x^{n}}^{(n)}\left\{W_{x^{n}}^{(n)}-e^{n a} W_{P^{(n)}}^{(n)}>0\right\}\right] .
$$

This leads to the implications:

$$
\begin{aligned}
a<\underline{I}(\overrightarrow{\boldsymbol{P}} \| \overrightarrow{\boldsymbol{W}}) & \Longrightarrow \lim _{n \rightarrow \infty} \sum_{x^{n}} P^{(n)}\left(x^{n}\right) \operatorname{Tr}\left[W_{x^{n}}^{(n)}\left\{W_{x^{n}}^{(n)}-e^{n a} W_{P^{(n)}}^{(n)}>0\right\}\right]=1 \\
& \Longrightarrow a \leq \liminf _{n \rightarrow \infty} \frac{1}{n} I\left(P^{(n)}, W^{(n)}\right),
\end{aligned}
$$

which proves the lemma.

Using this lemma and invoking that in the stationary memoryless case

$$
\sup _{P^{(n)} \in \mathcal{P}\left(\mathcal{X}^{n}\right)} I\left(P^{(n)}, W^{(n)}\right)=n \sup _{P \in \mathcal{P}(\mathcal{X})} I(P, W)
$$

we see that (36) follows from the general formula $C(\overrightarrow{\boldsymbol{W}}) \leq \max _{\overrightarrow{\boldsymbol{P}}} \underline{I}(\overrightarrow{\boldsymbol{P}}, \overrightarrow{\boldsymbol{W}})$.

Before proceeding to the direct and strong converse parts, we introduce quantum analogues of the spectral inf- and sup-divergence rates [6] (see Remark [6): given arbitrary sequences of states $\overrightarrow{\boldsymbol{\rho}}=\left\{\rho^{(n)}\right\}$ and $\overrightarrow{\boldsymbol{\sigma}}=\left\{\sigma^{(n)}\right\}$, let

$$
\begin{aligned}
& \bar{D}(\overrightarrow{\boldsymbol{\rho}} \| \overrightarrow{\boldsymbol{\sigma}}) \stackrel{\text { def }}{=} \inf \left\{a \mid \lim _{n \rightarrow \infty} \operatorname{Tr}\left[\rho^{(n)}\left\{\rho^{(n)}-e^{n a} \sigma^{(n)}>0\right\}\right]=0\right\}, \\
& \underline{D}(\overrightarrow{\boldsymbol{\rho}} \| \overrightarrow{\boldsymbol{\sigma}}) \stackrel{\text { def }}{=} \sup \left\{a \mid \lim _{n \rightarrow \infty} \operatorname{Tr}\left[\rho^{(n)}\left\{\rho^{(n)}-e^{n a} \sigma^{(n)}>0\right\}\right]=1\right\} .
\end{aligned}
$$


Note that $\underline{D}(\overrightarrow{\boldsymbol{\rho}} \| \overrightarrow{\boldsymbol{\sigma}}) \leq \bar{D}(\overrightarrow{\boldsymbol{\rho}} \| \overrightarrow{\boldsymbol{\sigma}})$ and that $\underline{D}(\overrightarrow{\boldsymbol{\rho}} \| \overrightarrow{\boldsymbol{\sigma}}) \leq \liminf _{n \rightarrow \infty} \frac{1}{n} D\left(\rho^{(n)} \| \sigma^{(n)}\right)$, the latter of which can be proved similarly to Lemma 5 . The following relation, which was shown in [10, will play an essential role in the later arguments: in the quantum i.i.d. case when $\overrightarrow{\boldsymbol{\rho}}=\left\{\rho^{\otimes n}\right\}_{n=1}^{\infty}$ and $\overrightarrow{\boldsymbol{\sigma}}=\left\{\sigma^{\otimes n}\right\}_{n=1}^{\infty}$, we have

$$
\bar{D}(\overrightarrow{\boldsymbol{\rho}} \| \overrightarrow{\boldsymbol{\sigma}})=\underline{D}(\overrightarrow{\boldsymbol{\rho}} \| \overrightarrow{\boldsymbol{\sigma}})=D(\rho \| \sigma) .
$$

Now let us observe how the direct part

$$
C(\overrightarrow{\boldsymbol{W}}) \geq \sup _{P \in \mathcal{P}(\mathcal{X})} I(P, W)
$$

follows from the general formula. Let $P$ be an arbitrary distribution in $\mathcal{P}(\mathcal{X})$ and $P^{(n)} \in \mathcal{P}\left(\mathcal{X}^{n}\right)$ be the $n$th i.i.d. extension: $P^{(n)}\left(x^{n}\right)=P\left(x_{1}\right) \cdots P\left(x_{n}\right)$ for $x^{n}=$ $\left(x_{1}, \ldots, x_{n}\right)$. Denoting the support of $P$ by $\left\{u_{1}, \ldots, u_{k}\right\} \subset \mathcal{X}$ and letting $\lambda_{i}=P\left(u_{i}\right)$, $\rho_{i}=W_{u_{i}}$ and $\sigma=W_{P}=\sum_{i} \lambda_{i} \rho_{i}$, we have

$$
\begin{aligned}
& \sum_{x^{n} \in \mathcal{X}^{(n)}} P^{(n)}\left(x^{n}\right) \operatorname{Tr}\left[W_{x^{n}}^{(n)}\left\{W_{x^{n}}^{(n)}-e^{n a} W_{P^{(n)}}^{(n)}>0\right\}\right] \\
= & \sum_{i_{1}, \ldots, i_{n}} \lambda_{i_{1}} \cdots \lambda_{i_{n}} \operatorname{Tr}\left[\left(\rho_{i_{1}} \otimes \cdots \otimes \rho_{i_{n}}\right)\left\{\rho_{i_{1}} \otimes \cdots \otimes \rho_{i_{n}}-e^{n a} \sigma^{\otimes n}>0\right\}\right] \\
= & \operatorname{Tr}\left[R^{\otimes n}\left\{R^{\otimes n}-e^{n a} S^{\otimes n}>0\right\}\right],
\end{aligned}
$$

where

$$
R \stackrel{\text { def }}{=}\left(\begin{array}{ccc}
\lambda_{1} \rho_{1} & & 0 \\
0 & \ddots & \lambda_{k} \rho_{k}
\end{array}\right), \quad S \stackrel{\text { def }}{=}\left(\begin{array}{ccc}
\lambda_{1} \sigma & & 0 \\
& \ddots & \\
0 & & \lambda_{k} \sigma
\end{array}\right) .
$$

We thus have for the sequences $\overrightarrow{\boldsymbol{P}}=\left\{P^{(n)}\right\}, \overrightarrow{\boldsymbol{R}}=\left\{R^{\otimes n}\right\}$ and $\overrightarrow{\boldsymbol{S}}=\left\{S^{\otimes n}\right\}$

$$
\underline{I}(\overrightarrow{\boldsymbol{P}}, \overrightarrow{\boldsymbol{W}})=\underline{D}(\overrightarrow{\boldsymbol{R}} \| \overrightarrow{\boldsymbol{S}})=D(R \| S)=I(P, W),
$$

where the second equality follows from (40) and the rest are immediate from the definitions of the quantities. This, combined with (7), completes the proof of (41).

Remark 14 Essential in the above derivation of (41) from (17) is the use of $\underline{D}(\overrightarrow{\boldsymbol{\rho}} \| \overrightarrow{\boldsymbol{\sigma}}) \geq$ $D(\rho \| \sigma)$ for sequences of i.i.d. states. The proof of the inequality given in [10] is based on the direct part of the quantum Stein's lemma for a hypothesis testing problem on $\rho^{\otimes n}$ and $\sigma^{\otimes n}$, which was first shown by Hiai and Petz [11, whereas the classical counterpart of the inequality is a direct consequence of the weak law of large numbers. Hence the above derivation can be thought of as a proof of the channel coding theorem via the theory of quantum hypothesis testing (cf. Remark [15 below). It should be noted that a significant characteristic of the proof lies in separation of 
the coding part and the limiting part; the former is entirely coped with in the general formula (17), or equivalently in the non-asymptotic arguments of Lemma 2 and Lemma 3. while the latter relies on the asymptotic analysis of quantum hypothesis testing. Another proof of (41) with a similar approach is found in [13, 14, where the coding part is proved by a variant of quantum Feinstein's lemma (cf. Remark 9) and the limiting part is based on an asymptotic analysis made in [27] (cf. Remark 17] below) on a variant of $\underline{D}(\overrightarrow{\boldsymbol{\rho}} \| \overrightarrow{\boldsymbol{\sigma}})$, which is much easier to treat than the original $\underline{D}(\overrightarrow{\boldsymbol{\rho}} \| \overrightarrow{\boldsymbol{\sigma}})$.

Remark 15 In an actual fact, (41) can be proved by directly applying Lemma 2 to the direct part of quantum Stein's lemma as follows, without appealing to the general formula (77). Given $P \in \mathcal{P}(\mathcal{X})$, let $R$ and $S$ be defined by (42), which can be represented as $R=\oplus_{x} P(x) W_{x}$ and $S=\oplus_{x} P(x) W_{P}$. For an arbitrary $\varepsilon>0$ and a sufficiently large $n$, it follows from the quantum Stein's lemma that there exists a projection of the form $T^{(n)}=\oplus_{x^{n}} T_{x^{n}}^{(n)}$, where $\left\{T_{x^{n}}^{(n)}\right\}$ are projections on $\mathcal{H}^{\otimes n}$, such that

$$
\begin{aligned}
& \operatorname{Tr}\left[R^{\otimes n} T^{(n)}\right]=\sum_{x^{n}} P^{(n)}\left(x^{n}\right) \operatorname{Tr}\left[W_{x^{n}}^{(n)} T_{x^{n}}^{(n)}\right] \geq 1-\varepsilon, \\
& \operatorname{Tr}\left[S^{\otimes n} T^{(n)}\right]=\sum_{x^{n}} P^{(n)}\left(x^{n}\right) \operatorname{Tr}\left[W_{P}^{\otimes n} T_{x^{n}}^{(n)}\right] \leq e^{-n(D(R \| S)-\varepsilon)} .
\end{aligned}
$$

Given an encoder $\varphi^{(n)}:\{1, \ldots, N\} \rightarrow \mathcal{X}^{n}$, define the decoding POVM $Z^{(n)}=$ $\left\{Z_{i}^{(n)}\right\}_{i=1}^{N}$ by

$$
Z_{i}^{(n)} \stackrel{\text { def }}{=}\left(\sum_{j=1}^{N} T_{\varphi^{(n)}(j)}^{(n)}\right)^{-\frac{1}{2}} T_{\varphi^{(n)}(i)}^{(n)}\left(\sum_{j=1}^{N} T_{\varphi^{(n)}(j)}^{(n)}\right)^{-\frac{1}{2}}
$$

Then replacing $Y^{(n)}$ with $Z^{(n)}$ in the proof of Lemma 3, using Lemma 2 for $c=1$ (e.g.) and applying the random coding with respect to $P^{(n)}$, we see that there exists a code $\Phi^{(n)}$ satisfying

$$
\begin{aligned}
\mathrm{P}_{\mathrm{e}}\left[\Phi^{(n)}\right] & \leq 2\left(1-\operatorname{Tr}\left[R^{\otimes n} T^{(n)}\right]\right)+4 N \operatorname{Tr}\left[S^{\otimes n} T^{(n)}\right] \\
& \leq 2 \varepsilon+4 e^{-n(D(R \| S)-\varepsilon)} N,
\end{aligned}
$$

which proves (41) by $D(R \| S)=I(P, W)$.

Remark 16 As is shown in section 4 of [11], from the fact that the (direct part of) quantum Stein's lemma holds for states on every finite-dimensional matrix algebra, it is immediately concluded that the lemma holds also for states on every AFD (approximately finite dimensional) operator algebra, including the algebra $B(\mathcal{H})$ of bounded operators on a separable Hilbert space $\mathcal{H}$. This means that our proof of (33) is valid for every channel $W$ on a separable Hilbert space $\mathcal{H}$ without the finiteness assumption (35). Note also that a similar argument based on the AFD property can be applied to the channel coding problem directly to remove the finiteness assumption from the proof of Holevo-Schumacher-Westmoreland. 
Remark 17 Combination of the argument in Remark [15] and the derivation of the direct part of quantum Stein's lemma given in [27] will provide one of the simplest proofs of (41) (for a finite-dimensional $\mathcal{H}$ ). In addition, application of Theorem 2 of [27] to (44) implies that for any $n$ and $a>0$ there exists a code $\Phi^{(n)}$ satisfying $\left|\Phi^{(n)}\right|=e^{n a}$ and

$$
\mathrm{P}_{\mathrm{e}}\left[\Phi^{(n)}\right] \leq 6(n+1)^{d} e^{-n \bar{\varphi}(a)}
$$

where $d \stackrel{\text { def }}{=} k \operatorname{dim} \mathcal{H}$ (the size of the matrices $R$ and $S$ ) and

$$
\begin{aligned}
\bar{\varphi}(a) & \stackrel{\text { def }}{=} \max _{0 \leq t \leq 1}\left(-a t-\log \operatorname{Tr}\left[R S^{\frac{t}{2}} R^{-t} S^{\frac{t}{2}}\right]\right) \\
& =\max _{0 \leq t \leq 1}\left(-a t-\log \sum_{i} \lambda_{i} \operatorname{Tr}\left[\rho_{i} \sigma^{\frac{t}{2}} \rho_{i}^{-t} \sigma^{\frac{t}{2}}\right]\right) .
\end{aligned}
$$

As was shown in [27], $\bar{\varphi}(a)>0$ holds for any $a<D(R \| S)=I(P, W)$, and (45) gives an exponential bound on the error probability.

Next we proceed to the strong converse part

$$
C^{\dagger}(\overrightarrow{\boldsymbol{W}}) \leq \sup _{P \in \mathcal{P}(\mathcal{X})} I(P, W)
$$

under the assumption that $\mathcal{H}$ is finite-dimensional. In order to link (46) to the general formula, we use the following relations ([28, 29]):

$$
\begin{aligned}
\sup _{P \in \mathcal{P}(\mathcal{X})} I(P, W) & =\sup _{P \in \mathcal{P}(\mathcal{X})} \min _{\sigma \in \mathcal{S}(\mathcal{H})} J(P, \sigma, W) \\
& =\min _{\sigma \in \mathcal{S}(\mathcal{H})} \sup _{P \in \mathcal{P}(\mathcal{X})} J(P, \sigma, W) \\
& =\min _{\sigma \in \mathcal{S}(\mathcal{H})} \sup _{x \in \mathcal{X}} D\left(W_{x} \| \sigma\right),
\end{aligned}
$$

where

$$
J(P, \sigma, W) \stackrel{\text { def }}{=} \sum_{x \in \mathcal{X}} P(x) D\left(W_{x} \| \sigma\right) .
$$

These relations can be derived just in parallel with its classical counter part (e.g., pp.142-147 of [30], Theorem 4.5.1 of [31]) by the use of a mini-max theorem for a certain class of two-variable convex-concave functions (e.g. Chap.VI of [32]), combined with the fact that the supremum of $\sup _{P \in \mathcal{P}(\mathcal{X})} I(P, W)$ can be attained when $\left\{W_{x} \mid x \in \mathcal{X}\right\}$ is closed $([18,26])$.

In proving the strong converse of the quantum hypothesis testing problem for two i.i.d. states, which is equivalent to the part $\bar{D}(\overrightarrow{\boldsymbol{\rho}} \| \overrightarrow{\boldsymbol{\sigma}}) \leq D(\rho \| \sigma)$ in (40) (see [10]), Ogawa and Nagaoka [12] showed that for any states $\rho, \sigma$ and any numbers $c>0$ and $0 \leq s \leq 1$

$$
\operatorname{Tr}[\rho\{\rho-c \sigma>0\}] \leq c^{-s} \operatorname{Tr}\left[\rho^{1+s} \sigma^{-s}\right] .
$$


Applying this to the states $W_{x^{n}}^{(n)}, \sigma^{\otimes n}$ and $c=e^{n a}$, we have

$$
\begin{aligned}
\operatorname{Tr}\left[W_{x^{n}}^{(n)}\left\{W_{x^{n}}^{(n)}-e^{n a} \sigma^{\otimes n}>0\right\}\right] & \leq \exp \left[-n\left(a s-\frac{1}{n} \sum_{i=1}^{n} \log \operatorname{Tr}\left[W_{x_{i}}^{1+s} \sigma^{-s}\right]\right)\right] \\
& \leq \exp \left[-n\left(a s-\sup _{x \in \mathcal{X}} \log \operatorname{Tr}\left[W_{x}^{1+s} \sigma^{-s}\right]\right)\right] .
\end{aligned}
$$

Now assume that $\operatorname{Im} \sigma \supset \operatorname{Im} W_{x}$ for all $x \in \mathcal{X}$, where $\operatorname{Im}$ denotes the image (range) of an operator, let $\bar{\Delta}$ be the closure of the range $\Delta=\left\{W_{x} \mid x \in \mathcal{X}\right\}$, and define the function $f:[0,1] \times \bar{\Delta} \rightarrow \mathbb{R}$ by $f(s, \rho)=\log \operatorname{Tr}\left[\rho^{1+s} \sigma^{-s}\right]$. Then we have $f(0, \rho)=0$ and

$$
\frac{\partial}{\partial s} f(0, \rho)=D(\rho \| \sigma)
$$

Moreover, since the derivative

$$
\frac{\partial}{\partial s} f(s, \rho)=\frac{\operatorname{Tr}\left[\rho^{1+s}(\log \rho-\log \sigma) \sigma^{-s}\right]}{\operatorname{Tr}\left[\rho^{1+s} \sigma^{-s}\right]}
$$

is continuous with respect to both $s$ and $\rho$, and since $\bar{\Delta}$ is compact, we see that the differentiation in (50) is uniform in $\rho$; i.e.,

$$
\lim _{s \downarrow} \max _{\rho \in \bar{\Delta}}\left|\frac{f(s, \rho)}{s}-D(\rho \| \sigma)\right|=0 .
$$

Let $a$ be an arbitrary number satisfying $a>\max _{\rho \in \bar{\Delta}} D(\rho \| \sigma)=\sup _{x \in \mathcal{X}} D\left(W_{x} \| \sigma\right)$. It then follows from the above uniform convergence that there exists an $s_{0}>0$ such that for any $0<s \leq s_{0}$

$$
a s>\max _{\rho \in \bar{\Delta}} f(s, \rho)=\sup _{x \in \mathcal{X}} \log \operatorname{Tr}\left[W_{x}^{1+s} \sigma^{-s}\right] .
$$

Invoking (49), this implies that for any sequence $\overrightarrow{\boldsymbol{x}}=\left\{x^{n}\right\} \in \overrightarrow{\mathcal{X}}$, where $\overrightarrow{\mathcal{X}}=\left\{\mathcal{X}^{n}\right\}$ is identified with the product set $\prod_{n} \mathcal{X}^{n}$, we have

$$
\lim _{n \rightarrow \infty} \operatorname{Tr}\left[W_{x^{n}}^{(n)}\left\{W_{x^{n}}^{(n)}-e^{n a} \sigma^{\otimes n}>0\right\}\right]=0 \quad \text { for } \forall a>\sup _{x \in \mathcal{X}} D\left(W_{x} \| \sigma\right),
$$

or equivalently

$$
\bar{D}\left(\overrightarrow{\boldsymbol{W}}_{\overrightarrow{\boldsymbol{x}}} \| \overrightarrow{\boldsymbol{\sigma}}\right) \leq \sup _{x \in \mathcal{X}} D\left(W_{x} \| \sigma\right)
$$

where $\overrightarrow{\boldsymbol{W}}_{\overrightarrow{\boldsymbol{x}}}=\left\{W_{x^{n}}^{(n)}\right\}$ and $\overrightarrow{\boldsymbol{\sigma}}=\left\{\sigma^{\otimes n}\right\}$. Although we assumed $\operatorname{Im} \sigma \supset \operatorname{Im} W_{x}, \forall x \in \mathcal{X}$ above, this inequality is valid for any $\sigma \in \mathcal{S}(\mathcal{H})$ because $\sup _{x \in \mathcal{X}} D\left(W_{x} \| \sigma\right)=\infty$ if 
$\operatorname{Im} \sigma \not \supset \operatorname{Im} W_{x}$ for some $x \in \mathcal{X}$. Now the desired inequality (46) is derived from the general formula (10) as follows:

$$
\begin{aligned}
C^{\dagger}(\overrightarrow{\boldsymbol{W}}) & =\max _{\overrightarrow{\boldsymbol{P}} \in \overrightarrow{\mathcal{P}}(\overrightarrow{\mathcal{X}})} \min _{\overrightarrow{\boldsymbol{\sigma}} \in \overrightarrow{\mathcal{S}}(\overrightarrow{\mathcal{H}})} \bar{J}(\overrightarrow{\boldsymbol{P}}, \overrightarrow{\boldsymbol{\sigma}}, \overrightarrow{\boldsymbol{W}}) \\
& \leq \min _{\overrightarrow{\boldsymbol{\sigma}} \in \overrightarrow{\mathcal{S}}(\overrightarrow{\mathcal{H}})} \max _{\overrightarrow{\boldsymbol{P}} \in \overrightarrow{\mathcal{P}}(\overrightarrow{\mathcal{X}})} \bar{J}(\overrightarrow{\boldsymbol{P}}, \overrightarrow{\boldsymbol{\sigma}}, \overrightarrow{\boldsymbol{W}}) \\
& =\min _{\overrightarrow{\boldsymbol{\sigma}} \in \overrightarrow{\mathcal{S}}(\overrightarrow{\mathcal{H}})} \max _{\overrightarrow{\boldsymbol{x}} \in \mathcal{\mathcal { X }}} \bar{D}\left(\overrightarrow{\boldsymbol{W}}_{\overrightarrow{\boldsymbol{x}}} \| \overrightarrow{\boldsymbol{\sigma}}\right) \\
& \leq \min _{\sigma \in \mathcal{S}(\mathcal{H})} \max _{\overrightarrow{\boldsymbol{x}} \in \overrightarrow{\mathcal{X}}} \bar{D}\left(\overrightarrow{\boldsymbol{W}}_{\overrightarrow{\boldsymbol{x}}} \| \overrightarrow{\boldsymbol{\sigma}}\right) \quad \text { with } \quad \overrightarrow{\boldsymbol{\sigma}}=\left\{\sigma^{\otimes n}\right\} \\
& \leq \min _{\sigma \in \mathcal{S}(\mathcal{H})} \sup _{x \in \mathcal{X}} D\left(W_{x} \| \sigma\right)=\sup _{P \in \mathcal{P}(\mathcal{X})} I(P, W),
\end{aligned}
$$

where the last equality follows from (47).

\section{On the Holevo-Schumacher-Westmoreland de- coder}

Let us return to the situation in the proof of Lemma 3 where a probability distribution $P^{(n)}$ and an encoder $\varphi^{(n)}:\{1, \ldots, N\} \rightarrow \mathcal{X}^{(n)}$ are given. Instead of $Y^{(n)}$ defined in (19), consider the following POVM $\widetilde{Y}^{(n)}$ :

$$
\widetilde{Y}_{i}^{(n)} \stackrel{\text { def }}{=}\left(\sum_{j=1}^{N} \tau \nu_{j} \tau\right)^{-\frac{1}{2}} \tau \nu_{i} \tau\left(\sum_{j=1}^{N} \tau \nu_{j} \tau\right)^{-\frac{1}{2}}
$$

where

$$
\tau \stackrel{\text { def }}{=}\left\{W_{P^{(n)}}^{(n)}<e^{-n b}\right\}, \quad \nu_{i} \stackrel{\text { def }}{=}\left\{W_{\varphi^{(n)}(i)}^{(n)}>e^{-n c}\right\} .
$$

This type of decoder was introduced by Holevo [1] and Schumacher-Westmoreland [2] in proving the direct part of the capacity theorem. Let us investigate this decoder, comparing it with our $Y_{i}^{(n)}$ defined by (19) and (20).

Remark 18 More precisely, the decoder treated in [1, 2] was defined by (53) with projections $\tau$ and $\nu_{i}$ of the form

$$
\tau \stackrel{\text { def }}{=}\left\{e^{-n b^{\prime}}<W_{P^{(n)}}^{(n)}<e^{-n b}\right\}, \quad \nu_{i} \stackrel{\text { def }}{=}\left\{e^{-n c}<W_{\varphi^{(n)}(i)}^{(n)}<e^{-n c^{\prime}}\right\}
$$

where we have used a slight extension of the notation in (15):

$$
\{\alpha<A<\beta\}=\sum_{i: \alpha<\lambda_{i}<\beta} E_{i} .
$$

However, the asymptotic performance of the decoder does not depend on the particular values of $b^{\prime}, c^{\prime}$ as far as $b^{\prime}$ is sufficiently large and $c^{\prime}$ is sufficiently small. Hence we set $b^{\prime}=\infty$ and $c^{\prime}=-\infty$ to simplify the arguments. 
The authors of 1, 2] showed by a rather complicated calculation that the average error probability of the code $\widetilde{\Phi}^{(n)}=\left(N, \varphi^{(n)}, \widetilde{Y}^{(n)}\right)$ satisfies

$\mathrm{P}_{\mathrm{e}}\left[\widetilde{\Phi}^{(n)}\right] \leq \frac{1}{N} \sum_{i=1}^{N}\left\{3 \operatorname{Tr}\left[W_{\varphi^{(n)}(i)}^{(n)}(I-\tau)\right]+\operatorname{Tr}\left[W_{\varphi^{(n)}(i)}^{(n)}\left(I-\nu_{i}\right)\right]+\sum_{j(\neq i)} \operatorname{Tr}\left[W_{\varphi^{(n)}(i)}^{(n)} \tau \nu_{j} \tau\right]\right\}$.

Note that a simplified derivation of the inequality with slightly different coefficients was shown in [16]. Applying the random coding with respect to $P^{(n)}$ to (54) and noting that

$$
\begin{aligned}
& \operatorname{Tr}\left[W_{P^{(n)}}^{(n)}\left\{W_{P^{(n)}}^{(n)}<e^{-n b}\right\}\left\{W_{x^{n}}^{(n)}>e^{-n c}\right\}\left\{W_{P^{(n)}}^{(n)}<e^{-n b}\right\}\right] \\
& \leq\left\|W_{P^{(n)}}^{(n)}\left\{W_{P^{(n)}}^{(n)}<e^{-n b}\right\}\right\| \cdot \operatorname{Tr}\left[\left\{W_{x^{n}}^{(n)}>e^{-n c}\right\}\right] \\
& \leq e^{-n(b-c)},
\end{aligned}
$$

where $\|\cdot\|$ denotes the operator norm, we see that there exists a code $\Phi^{(n)}$ such that

$$
\begin{aligned}
\mathrm{P}_{\mathrm{e}}\left[\Phi^{(n)}\right] \leq & \operatorname{Tr}\left[W_{P^{(n)}}^{(n)}\left\{W_{P^{(n)}}^{(n)} \geq e^{-n b}\right\}\right]+\sum_{x^{n}} P^{(n)}\left(x^{n}\right) \operatorname{Tr}\left[W_{x^{n}}^{(n)}\left\{W_{x^{n}}^{(n)} \leq e^{-n c}\right\}\right] \\
& +e^{-n(b-c)} N .
\end{aligned}
$$

Now, for an arbitrary $\overrightarrow{\boldsymbol{P}}=\left\{P^{(n)}\right\} \in \overrightarrow{\mathcal{P}}(\overrightarrow{\mathcal{X}})$ let

$$
\begin{gathered}
\underline{H}\left(\overrightarrow{\boldsymbol{W}}_{\overrightarrow{\boldsymbol{P}}}\right) \stackrel{\text { def }}{=} \sup \left\{b \mid \lim _{n \rightarrow \infty} \operatorname{Tr}\left[W_{P^{(n)}}^{(n)}\left\{W_{P^{(n)}}^{(n)} \geq e^{-n b}\right\}\right]=0\right\} \\
=\sup \left\{b \mid \lim _{n \rightarrow \infty} \operatorname{Tr}\left[W_{P(n)}^{(n)}\left\{-\frac{1}{n} \log W_{P^{(n)}}^{(n)} \leq b\right\}\right]=0\right\}, \\
\bar{H}(\overrightarrow{\boldsymbol{W}} \mid \overrightarrow{\boldsymbol{P}}) \stackrel{\text { def }}{=} \inf \left\{c \mid \lim _{n \rightarrow \infty} \sum_{x^{n}} P^{(n)}\left(x^{n}\right) \operatorname{Tr}\left[W_{x^{n}}^{(n)}\left\{W_{x^{n}}^{(n)} \leq e^{-n c}\right\}\right]=0\right\} \\
=\inf \left\{c \mid \lim _{n \rightarrow \infty} \sum_{x^{n}} P^{(n)}\left(x^{n}\right) \operatorname{Tr}\left[W_{x^{n}}^{(n)}\left\{-\frac{1}{n} \log W_{x^{n}}^{(n)} \geq c\right\}\right]=0\right\},
\end{gathered}
$$

and assume that $\bar{H}(\overrightarrow{\boldsymbol{W}} \mid \overrightarrow{\boldsymbol{P}})<\infty$. It then follows from (56) that there exists a sequence of codes $\overrightarrow{\boldsymbol{\Phi}}=\left\{\Phi^{(n)}\right\}$ such that $\lim _{n \rightarrow \infty} \mathrm{P}_{\mathrm{e}}\left[\Phi^{(n)}\right]=0$ with the rate $\liminf _{n \rightarrow \infty} \frac{1}{n} \log \left|\Phi^{(n)}\right|$ being arbitrarily close to $\underline{H}\left(\overrightarrow{\boldsymbol{W}}_{\overrightarrow{\boldsymbol{P}}}\right)-\bar{H}(\overrightarrow{\boldsymbol{W}} \mid \overrightarrow{\boldsymbol{P}})$; i.e., we have

$$
C(\overrightarrow{\boldsymbol{W}}) \geq \max _{\overrightarrow{\boldsymbol{P}} \in \overrightarrow{\mathcal{P}}(\overrightarrow{\mathcal{X}})}\left\{\underline{H}\left(\overrightarrow{\boldsymbol{W}}_{\overrightarrow{\boldsymbol{P}}}\right)-\bar{H}(\overrightarrow{\boldsymbol{W}} \mid \overrightarrow{\boldsymbol{P}}) \mid \bar{H}(\overrightarrow{\boldsymbol{W}} \mid \overrightarrow{\boldsymbol{P}})<\infty\right\} .
$$

The quantities $\underline{H}\left(\overrightarrow{\boldsymbol{W}}_{\overrightarrow{\boldsymbol{P}}}\right)$ and $\bar{H}(\overrightarrow{\boldsymbol{W}} \mid \overrightarrow{\boldsymbol{P}})$ are regarded as information-spectrum analogues of the von Neumann entropy and its conditional version. Indeed, for a 
stationary memoryless channel $W_{x^{n}}^{(n)}=W_{x_{1}} \otimes \cdots \otimes W_{x_{n}}$ with i.i.d. $\quad P^{(n)}\left(x^{n}\right)=$ $P\left(x_{1}\right) \cdots P\left(x_{n}\right)$ the law of large numbers yields

$$
\begin{aligned}
\underline{H}\left(\overrightarrow{\boldsymbol{W}}_{\overrightarrow{\boldsymbol{P}}}\right) & =H\left(W_{P}\right)=-\operatorname{Tr}\left[W_{P} \log W_{P}\right], \\
\bar{H}(\overrightarrow{\boldsymbol{W}} \mid \overrightarrow{\boldsymbol{P}}) & =H(W \mid P) \stackrel{\text { def }}{=} \sum_{x} P(x) H\left(W_{x}\right),
\end{aligned}
$$

which leads to $C(\overrightarrow{\boldsymbol{W}}) \geq \sup _{P \in \mathcal{P}(\mathcal{X})}\left(H\left(W_{P}\right)-H(W \mid P)\right)=\sup _{P \in \mathcal{P}(\mathcal{X})} I(P, W)$ under the finiteness assumption (35) (cf. Remark 16). This is just what was shown in [1, 2].

Remark 19 Inequality (15) of Lemma2 2 can be applied to the code $\widetilde{\Phi}^{(n)}=\left(N, \varphi^{(n)}, \widetilde{Y}^{(n)}\right)$ to derive (57) more straightforwardly than the derivations in [1, 2, 16. Indeed, letting $c=1$ (e.g.) in (15) we have

$$
\begin{aligned}
\mathrm{P}_{\mathrm{e}}\left[\widetilde{\Phi}^{(n)}\right] & \leq \frac{1}{N} \sum_{i=1}^{N}\left\{2 \operatorname{Tr}\left[W_{\varphi^{(n)}(i)}^{(n)}\left(I-\tau \nu_{i} \tau\right)\right]+4 \sum_{j \neq i} \operatorname{Tr}\left[W_{\varphi^{(n)}(i)}^{(n)} \tau \nu_{j} \tau\right]\right\} \\
& \leq \frac{1}{N} \sum_{i=1}^{N}\left\{4 \operatorname{Tr}\left[W_{\varphi^{(n)}(i)}^{(n)}(I-\tau)\right]+2 \operatorname{Tr}\left[W_{\varphi^{(n)}(i)}^{(n)}\left(I-\nu_{i}\right)\right]+4 \sum_{j(\neq i)} \operatorname{Tr}\left[W_{\varphi^{(n)}(i)}^{(n)} \tau \nu_{j} \tau\right]\right\}
\end{aligned}
$$

where the second inequality follows from the next lemma. This leads to (57) as well as from (54).

Lemma 6 For any state $\rho$ and any projections $\nu, \tau$ such that $[\rho, \nu]=0$, we have

$$
\operatorname{Tr}[\rho \tau \nu \tau] \geq \operatorname{Tr}[\rho \nu]-2 \operatorname{Tr}[\rho(I-\tau)]
$$

Proof: Obvious from $0 \leq(I-\tau) \nu(I-\tau)=\tau \nu \tau-\nu+(I-\tau) \nu+\nu(I-\tau)$ and $\rho \nu=\nu \rho \leq \rho$.

Comparing (57) with (71) it is immediate that

$$
\max _{\overrightarrow{\boldsymbol{P}}} \underline{I}(\overrightarrow{\boldsymbol{P}}, \overrightarrow{\boldsymbol{W}}) \geq \max _{\overrightarrow{\boldsymbol{P}}}\left\{\underline{H}\left(\overrightarrow{\boldsymbol{W}}_{\overrightarrow{\boldsymbol{P}}}\right)-\bar{H}(\overrightarrow{\boldsymbol{W}} \mid \overrightarrow{\boldsymbol{P}}) \mid \bar{H}(\overrightarrow{\boldsymbol{W}} \mid \overrightarrow{\boldsymbol{P}})<\infty\right\} .
$$

Actually, a slightly stronger assertion holds:

Theorem 3 For every $\overrightarrow{\boldsymbol{P}} \in \overrightarrow{\mathcal{P}}(\overrightarrow{\mathcal{H}})$ with $\bar{H}(\overrightarrow{\boldsymbol{W}} \mid \overrightarrow{\boldsymbol{P}})<\infty$ we have

$$
\underline{I}(\overrightarrow{\boldsymbol{P}}, \overrightarrow{\boldsymbol{W}}) \geq \underline{H}\left(\overrightarrow{\boldsymbol{W}}_{\overrightarrow{\boldsymbol{P}}}\right)-\bar{H}(\overrightarrow{\boldsymbol{W}} \mid \overrightarrow{\boldsymbol{P}}) .
$$

Proof: It suffices to show that for any $b<\underline{H}\left(\overrightarrow{\boldsymbol{W}}_{\overrightarrow{\boldsymbol{P}}}\right), c>\bar{H}(\overrightarrow{\boldsymbol{W}} \mid \overrightarrow{\boldsymbol{P}})$ and $\varepsilon>0$ we have $\underline{I}(\overrightarrow{\boldsymbol{P}}, \overrightarrow{\boldsymbol{W}}) \geq b-c-\varepsilon$, or, equivalently that if

$$
\lim _{n \rightarrow \infty} \operatorname{Tr}\left[W_{P(n)}^{(n)}\left\{W_{P(n)}^{(n)} \geq e^{-n b}\right\}\right]=0
$$


and

$$
\lim _{n \rightarrow \infty} \sum_{x^{n} \in \mathcal{X}^{(n)}} P^{(n)}\left(x^{n}\right) \operatorname{Tr}\left[W_{x^{n}}^{(n)}\left\{W_{x^{n}}^{(n)}>e^{-n c}\right\}\right]=1
$$

then

$$
\lim _{n \rightarrow \infty} \sum_{x^{n} \in \mathcal{X}^{(n)}} P^{(n)}\left(x^{n}\right) \operatorname{Tr}\left[W_{x^{n}}^{(n)}\left\{W_{x^{n}}^{(n)}-e^{n(b-c-\varepsilon)} W_{P^{(n)}}^{(n)}>0\right\}\right]=1 .
$$

We obtain

$$
\begin{aligned}
& \sum_{x^{n} \in \mathcal{X}^{(n)}} P^{(n)}\left(x^{n}\right) \operatorname{Tr}\left[W_{x^{n}}^{(n)}\left\{W_{x^{n}}^{(n)}-e^{n(b-c-\varepsilon)} W_{P^{(n)}}^{(n)}>0\right\}\right] \\
\geq & \sum_{x^{n} \in \mathcal{X}^{(n)}} P^{(n)}\left(x^{n}\right) \operatorname{Tr}\left[\left(W_{x^{n}}^{(n)}-e^{n(b-c-\varepsilon)} W_{P^{(n)}}^{(n)}\right)\left\{W_{x^{n}}^{(n)}-e^{n(b-c-\varepsilon)} W_{P^{(n)}}^{(n)}>0\right\}\right] \\
\geq & \sum_{x^{n} \in \mathcal{X}^{(n)}} P^{(n)}\left(x^{n}\right) \operatorname{Tr}\left[\left(W_{x^{n}}^{(n)}-e^{n(b-c-\varepsilon)} W_{P^{(n)}}^{(n)}\right)\left\{W_{P^{(n)}}^{(n)}<e^{-n b}\right\}\left\{W_{x^{n}}^{(n)}>e^{-n c}\right\}\left\{W_{P^{(n)}}^{(n)}<e^{-n b}\right\}\right] \\
\geq & \sum_{x^{n} \in \mathcal{X}^{(n)}} P^{(n)}\left(x^{n}\right) \operatorname{Tr}\left[W_{x^{n}}^{(n)}\left\{W_{x^{n}}^{(n)}>e^{-n c}\right\}\right]-2 \sum_{x^{n} \in \mathcal{X}^{(n)}} P^{(n)}\left(x^{n}\right) \operatorname{Tr}\left[W_{x^{n}}^{(n)}\left\{W_{P^{(n)}}^{(n)} \geq e^{-n b}\right\}\right] \\
& -e^{n(b-c-\varepsilon)} \sum_{x^{n} \in \mathcal{X}^{(n)}} P^{(n)}\left(x^{n}\right) \operatorname{Tr}\left[W_{P^{(n)}}^{(n)}\left\{W_{P^{(n)}}^{(n)}<e^{-n b}\right\}\left\{W_{x^{n}}^{(n)}>e^{-n c}\right\}\right] \\
\geq & \sum_{x^{n} \in \mathcal{X}^{(n)}} P^{(n)}\left(x^{n}\right) \operatorname{Tr}\left[W_{x^{n}}^{(n)}\left\{W_{x^{n}}^{(n)}>e^{-n c}\right\}\right]-2 \operatorname{Tr}\left[W_{P^{(n)}}^{(n)}\left\{W_{P^{(n)}}^{(n)} \geq e^{-n b}\right\}\right]-e^{-n \varepsilon},
\end{aligned}
$$

where the second inequality follows from (25), the third from Lemma 6 and the last from (55). Now it is clear that (62) and (61) implies (63).

Remark 20 Theorem 3 enables us to derive the direct part (41) for a stationary memoryless channel from the general formula (17) via equations (58) and (59). This is essentially equivalent to the simplification of Holevo-Schumacher-Westmoreland's proof explained in Remark [19, but can also be regarded as a variation of the scenario of section [6 to derive (41) from (7) via $\underline{D}(\overrightarrow{\boldsymbol{\rho}} \| \overrightarrow{\boldsymbol{\sigma}}) \geq D(\rho \| \sigma)$ for $\overrightarrow{\boldsymbol{\rho}}=\left\{\rho^{\otimes n}\right\}_{n=1}^{\infty}$ and $\overrightarrow{\boldsymbol{\sigma}}=\left\{\sigma^{\otimes n}\right\}_{n=1}^{\infty}$. That is, just in parallel with the proof of Theorem [3], we can show for any sequences of states $\overrightarrow{\boldsymbol{\rho}}=\left\{\rho^{(n)}\right\}$ and $\overrightarrow{\boldsymbol{\sigma}}=\left\{\sigma^{(n)}\right\}$ that

$$
\underline{D}(\overrightarrow{\boldsymbol{\rho}} \| \overrightarrow{\boldsymbol{\sigma}}) \geq \underline{K}(\overrightarrow{\boldsymbol{\rho}} \| \overrightarrow{\boldsymbol{\sigma}})-\bar{H}(\overrightarrow{\boldsymbol{\rho}}),
$$

where

$$
\begin{gathered}
\underline{K}(\overrightarrow{\boldsymbol{\rho}} \| \overrightarrow{\boldsymbol{\sigma}}) \stackrel{\text { def }}{=} \sup \left\{b \mid \lim _{n \rightarrow \infty} \operatorname{Tr}\left[\rho^{(n)}\left\{-\frac{1}{n} \log \sigma^{(n)} \leq b\right\}\right]=0\right\}, \\
\bar{H}(\overrightarrow{\boldsymbol{\rho}}) \stackrel{\text { def }}{=} \inf \left\{c \mid \lim _{n \rightarrow \infty} \operatorname{Tr}\left[\rho^{(n)}\left\{-\frac{1}{n} \log \rho^{(n)} \geq c\right\}\right]=0\right\},
\end{gathered}
$$


which yields for $\overrightarrow{\boldsymbol{\rho}}=\left\{\rho^{\otimes n}\right\}_{n=1}^{\infty}$ and $\overrightarrow{\boldsymbol{\sigma}}=\left\{\sigma^{\otimes n}\right\}_{n=1}^{\infty}$ that $\underline{D}(\overrightarrow{\boldsymbol{\rho}} \| \overrightarrow{\boldsymbol{\sigma}}) \geq-\operatorname{Tr}[\rho \log \sigma]-$ $H(\rho)=D(\rho \| \sigma)$. Combination of this argument, which provides another simple proof of the direct part of the quantum Stein's lemma (cf. [10]), with the scenario of section [6] is equivalent to the direct use of Theorem 3 mentioned above.

Remark 21 The classical counterpart of (60) is rather obvious (cf. Remark 6) :

$$
\begin{aligned}
\underline{I}(\mathbf{X} ; \mathbf{Y}) & =\mathrm{p}-\liminf _{n \rightarrow \infty} \frac{1}{n} \log \frac{W^{(n)}\left(Y^{(n)} \mid X^{(n)}\right)}{P_{Y^{(n)}}\left(Y^{(n)}\right)} \\
& \geq \mathrm{p}-\liminf _{n \rightarrow \infty} \frac{1}{n} \log \frac{1}{P_{Y^{(n)}}\left(Y^{(n)}\right)}-\mathrm{p}-\limsup _{n \rightarrow \infty} \frac{1}{n} \log \frac{1}{W^{(n)}\left(Y^{(n)} \mid X^{(n)}\right)} \\
& =\underline{H}(\mathbf{Y})-\bar{H}(\mathbf{Y} \mid \mathbf{X}) .
\end{aligned}
$$

\section{Capacity under cost constraint}

The cost constraint problem in the general setting is trivial as in the case of classical information spectrum methods [6]. Namely, given a sequence $\overrightarrow{\boldsymbol{W}}=\left\{W^{(n)}\right\}$ of channels $W^{(n)}: \mathcal{X}^{(n)} \rightarrow \mathcal{S}\left(\mathcal{H}^{(n)}\right)$ as well as a sequence $\overrightarrow{\boldsymbol{c}}=\left\{c^{(n)}\right\}$ of functions $c^{(n)}: \mathcal{X}^{(n)} \rightarrow \mathbb{R}$, which are called cost functions, and a real number $\gamma$, the capacity under cost constraint is nothing but the capacity $C\left(\overrightarrow{\boldsymbol{W}} \uparrow_{\overrightarrow{\boldsymbol{c}}, \gamma}\right)$ of the sequence of channels $\overrightarrow{\boldsymbol{W}} \uparrow_{\overrightarrow{\boldsymbol{c}}, \gamma}=\left\{W^{(n)} \uparrow_{c^{(n)}, \gamma}\right\}$, where $W^{(n)} \uparrow_{c^{(n)}, \gamma}$ is the restriction $W^{(n)} \uparrow_{c^{(n)}, \gamma}: \mathcal{X}_{c^{(n)}, \gamma}^{(n)} \ni x^{n} \mapsto W_{x^{n}}^{(n)}$ of the original channel $W^{(n)}$ to

$$
\mathcal{X}_{c^{(n)}, \gamma}^{(n)} \stackrel{\text { def }}{=}\left\{x^{n} \in \mathcal{X}^{(n)} \mid c^{(n)}\left(x^{n}\right) \leq n \gamma\right\}
$$

In addition, the strong converse property in this case is represented as $C\left(\overrightarrow{\boldsymbol{W}} \uparrow_{\overrightarrow{\boldsymbol{c}}, \gamma}\right)=$ $\left.C^{\dagger}(\overrightarrow{\boldsymbol{W}}\rceil_{\overrightarrow{\boldsymbol{c}}, \gamma}\right)$. Needless to say, we can apply the general formulas in Theorem 1 to these quantities.

Now let us consider the situation where $\overrightarrow{\boldsymbol{W}}=\left\{W^{(n)}\right\}$ is the stationary memoryless extension (32) of $W: \mathcal{X} \rightarrow \mathcal{S}(\mathcal{H})$ and $\overrightarrow{\boldsymbol{c}}=\left\{c^{(n)}\right\}$ is the additive extension

$$
c^{(n)}\left(x^{n}\right)=\sum_{i=1}^{n} c\left(x_{i}\right),
$$

where $c$ is a function $\mathcal{X} \rightarrow \mathbb{R}$. We shall prove the following theorem, which was essentially obtained by Holevo [16, 33. except for the strong converse part.

Theorem 4 In the stationary memoryless case with the additive cost, we have

$$
C\left(\overrightarrow{\boldsymbol{W}} \uparrow_{\overrightarrow{\boldsymbol{c}}, \gamma}\right)=\sup _{P \in \mathcal{P}_{c, \gamma}(\mathcal{X})} I(P, W)
$$

where

$$
\mathcal{P}_{c, \gamma}(\mathcal{X}) \stackrel{\text { def }}{=}\left\{P \in \mathcal{P}(\mathcal{X}) \mid E_{P}[c] \stackrel{\text { def }}{=} \sum_{x \in \mathcal{X}} P(x) c(x) \leq \gamma\right\} .
$$

If, in addition, $\operatorname{dim} \mathcal{H}<\infty$ then the strong converse holds: $C^{\dagger}\left(\overrightarrow{\boldsymbol{W}} \uparrow_{\overrightarrow{\boldsymbol{c}}, \gamma}\right)=C\left(\overrightarrow{\boldsymbol{W}}\left\lceil_{\overrightarrow{\boldsymbol{c}}, \gamma}\right)\right.$. 
We first show that the (weak) converse part

$$
\left.C(\overrightarrow{\boldsymbol{W}}\rceil_{\overrightarrow{\boldsymbol{c}}, \gamma}\right) \leq \sup _{P \in \mathcal{P}_{c, \gamma}(\mathcal{X})} I(P, W)
$$

is derived from the general formula. Let $\mathcal{P}^{(n)} \stackrel{\text { def }}{=} \mathcal{P}\left(\mathcal{X}_{c^{(n)}, \gamma}^{(n)}\right)$ be the totality of probability distributions on $\mathcal{X}^{(n)}=\mathcal{X}^{n}$ whose supports are finite subsets of

$$
\hat{\mathcal{X}}^{(n)} \stackrel{\text { def }}{=} \mathcal{X}_{c^{(n)}, \gamma}^{(n)}=\left\{\left(x_{1}, \ldots, x_{n}\right) \in \mathcal{X}^{n} \mid \frac{1}{n} \sum_{i=1}^{n} c\left(x_{i}\right) \leq \gamma\right\}
$$

For any $P^{(n)} \in \mathcal{P}^{(n)}$ and any permutation $\pi$ on $\{1, \ldots, n\}, P_{\pi}^{(n)}$ defined by $P_{\pi}^{(n)}\left(x_{1}, \ldots, x_{n}\right)$ $=P^{(n)}\left(x_{\pi(1)}, \ldots, x_{\pi(n)}\right)$ also belongs to $\mathcal{P}^{(n)}$ and satisfies $I\left(P^{(n)}, W^{(n)}\right)=I\left(P_{\pi}^{(n)}, W^{(n)}\right)$. Since $I\left(P^{(n)}, W^{(n)}\right)$ is concave with respect to $P^{(n)}$, we can restrict ourselves to symmetric distributions when considering $\sup _{P^{(n)} \in \mathcal{P}^{(n)}} I\left(P^{(n)}, W^{(n)}\right)$. For a symmetric $P^{(n)} \in \mathcal{P}^{(n)}$, the marginal distribution on $\mathcal{X}$ belongs to $\mathcal{P}_{c, \gamma}$ and satisfies $I\left(P^{(n)}, W^{(n)}\right) \leq n I(P, W)$. Hence we have

$$
\sup _{P^{(n)} \in \mathcal{P}^{(n)}} I\left(P^{(n)}, W^{(n)}\right) \leq n \sup _{P \in \mathcal{P}_{c, \gamma}} I(P, W),
$$

and (66) follows from Lemma 5] and (17) as in the costless case.

Next, let us consider the direct part

$$
C\left(\overrightarrow{\boldsymbol{W}} \uparrow_{\overrightarrow{\boldsymbol{c}}, \gamma}\right) \geq \sup _{P \in \mathcal{P}_{c, \gamma}(\mathcal{X})} I(P, W) .
$$

We use a slight modification of Lemma 3 as follows. Let $P$ be a probability distribution in $\mathcal{P}_{c, \gamma}(\mathcal{X})$ and $a$ be a real number. Given an arbitrary encoder $\varphi^{(n)}$ : $\{1, \ldots, N\} \rightarrow \mathcal{X}^{n}$, let the decoder $Y^{(n)}=\left\{Y_{1}^{(n)}, \ldots, Y_{N}^{(n)}\right\}$ be defined by

$$
Y_{i}^{(n)}=\left(\sum_{j=1}^{N} \pi_{j}\right)^{-\frac{1}{2}} \pi_{i}\left(\sum_{j=1}^{N} \pi_{j}\right)^{-\frac{1}{2}}
$$

where $\pi_{i} \stackrel{\text { def }}{=}\left\{W_{\varphi^{(n)}(i)}^{(n)}-e^{n a} W_{P}^{\otimes n}>0\right\}$. It then follows from Lemma 2 for $c=1$ (e.g.) that the average error probability of the code $\Phi^{(n)}=\left(N, \varphi^{(n)}, Y^{(n)}\right)$ is bounded by

$$
\mathrm{P}_{\mathrm{e}}\left[\Phi^{(n)}\right] \leq \frac{2}{N} \sum_{i=1}^{N} \operatorname{Tr}\left[W_{\varphi^{(n)}(i)}^{(n)}\left(I-\pi_{i}\right)\right]+\frac{4}{N} \sum_{i=1}^{N} \sum_{j \neq i} \operatorname{Tr}\left[W_{\varphi^{(n)}(i)}^{(n)} \pi_{j}\right]
$$

Now let $P^{(n)}$ be the $n$th i.i.d. extension of $P$ and $\hat{P}^{(n)} \in \mathcal{P}\left(\hat{\mathcal{X}}^{(n)}\right)$ be defined by

$$
\hat{P}^{(n)}\left(x^{n}\right)=P^{(n)}\left(x^{n}\right) / K_{n} \quad \text { for } \quad x^{n} \in \hat{\mathcal{X}}^{(n)}=\mathcal{X}_{c^{(n)}, \gamma}^{(n)},
$$


where $K_{n} \stackrel{\text { def }}{=} P^{(n)}\left(\hat{\mathcal{X}}^{(n)}\right)$. Note that due to the assumption $P \in \mathcal{P}_{c, \gamma}(\mathcal{X})$ and to the central limiting theorem we have

$$
\lim _{n \rightarrow \infty} K_{n} \geq \lim _{n \rightarrow \infty} P^{(n)}\left(\mathcal{X}_{c^{(n)}, E_{P}[c]}^{(n)}\right)=\frac{1}{2}
$$

Generating the encoder $\varphi^{(n)}$ randomly according to the distribution

$$
P_{\mathrm{rc}}^{(n)}\left(\varphi^{(n)}\right)=\hat{P}^{(n)}\left(\varphi^{(n)}(1)\right) \cdots \hat{P}^{(n)}\left(\varphi^{(n)}(N)\right),
$$

we see that there exists a code $\Phi^{(n)}$ for $W^{(n)} \uparrow_{c^{(n)}, \gamma}$ of size $N$ satisfying

$$
\begin{aligned}
\mathrm{P}_{\mathrm{e}}\left[\Phi^{(n)}\right] & \leq 2 \sum_{x^{n} \in \hat{\mathcal{X}}^{(n)}} \hat{P}^{(n)}\left(x^{n}\right) \operatorname{Tr}\left[W_{x^{n}}^{(n)}\left\{W_{x^{n}}^{(n)}-e^{n a} W_{P}^{\otimes n} \leq 0\right\}\right] \\
& +4 N \sum_{x^{n} \in \hat{\mathcal{X}}^{(n)}} \hat{P}^{(n)}\left(x^{n}\right) \operatorname{Tr}\left[\left(\sum_{x^{n} \in \hat{\mathcal{X}}^{(n)}} \hat{P}^{(n)}\left(x^{n^{\prime}}\right) W_{x^{n \prime}}^{(n)}\right)\left\{W_{x^{n}}^{(n)}-e^{n a} W_{P}^{\otimes n}>0\right\}\right] \\
& \leq \frac{2}{K_{n}} \sum_{x^{n} \in \hat{\mathcal{X}}^{(n)}} P^{(n)}\left(x^{n}\right) \operatorname{Tr}\left[W_{x^{n}}^{(n)}\left\{W_{x^{n}}^{(n)}-e^{n a} W_{P}^{\otimes n} \leq 0\right\}\right] \\
& +4 N \sum_{x^{n} \in \hat{\mathcal{X}}^{(n)}} \hat{P}^{(n)}\left(x^{n}\right) \operatorname{Tr}\left[\left(\frac{1}{K_{n}} W_{P}^{\otimes n}\right)\left\{W_{x^{n}}-e^{n a} W_{P}^{\otimes n}>0\right\}\right] \\
& \leq \frac{2}{K_{n}} \sum_{x^{n} \in \mathcal{X}^{n}} P^{(n)}\left(x^{n}\right) \operatorname{Tr}\left[W_{x^{n}}\left\{W_{x^{n}}-e^{n a} W_{P}^{\otimes n} \leq 0\right\}\right]+\frac{4}{K_{n}} N e^{-n a} .
\end{aligned}
$$

Thus, letting $\overrightarrow{\boldsymbol{P}}=\left\{P^{(n)}\right\}$ and recalling (69) we have

$$
C\left(\overrightarrow{\boldsymbol{W}} \uparrow_{\overrightarrow{\boldsymbol{c}}, \gamma}\right) \geq \underline{I}(\overrightarrow{\boldsymbol{P}}, \overrightarrow{\boldsymbol{W}})=I(P, W),
$$

where the last equality follows from (43). We have thus proved (67).

Remark 22 For the sequence $\overrightarrow{\hat{\boldsymbol{P}}}=\left\{\hat{P}^{(n)}\right\}$ defined from a $P \in \mathcal{P}_{c, \gamma}(\mathcal{X})$ by (68), the general formula (7) implies that

$$
\begin{aligned}
C\left(\overrightarrow{\boldsymbol{W}} \uparrow_{\overrightarrow{\boldsymbol{c}}, \gamma}\right) & \geq \underline{I}(\overrightarrow{\hat{\boldsymbol{P}}}, \overrightarrow{\boldsymbol{W}}) \\
& =\sup \left\{a \mid \lim _{n \rightarrow \infty} \sum_{x^{n} \in \hat{\mathcal{X}}^{(n)}} \hat{P}^{(n)}\left(x^{n}\right) \operatorname{Tr}\left[W_{x^{n}}^{(n)}\left\{W_{x^{n}}^{(n)}-e^{n a} W_{\hat{P}^{(n)}}^{(n)} \leq 0\right\}\right]=0\right\} \\
& \geq \sup \left\{a \mid \lim _{n \rightarrow \infty} \sum_{x^{n} \in \mathcal{X}^{(n)}} P^{(n)}\left(x^{n}\right) \operatorname{Tr}\left[W_{x^{n}}^{(n)}\left\{W_{x^{n}}^{(n)}-e^{n a} W_{\hat{P}^{(n)}}^{(n)} \leq 0\right\}\right]=0\right\} \\
& =\underline{J}(\overrightarrow{\boldsymbol{P}}, \overrightarrow{\boldsymbol{\sigma}}, \overrightarrow{\boldsymbol{W}}) \text { for } \overrightarrow{\boldsymbol{P}}=\left\{P^{(n)}\right\} \text { and } \overrightarrow{\boldsymbol{\sigma}}=\left\{W_{\hat{P}^{(n)}}^{(n)}\right\},
\end{aligned}
$$

where the second inequality follows from (69). So, if we could use $\underline{I}(\overrightarrow{\boldsymbol{P}}, \overrightarrow{\boldsymbol{W}})=$ $\min _{\overrightarrow{\boldsymbol{\sigma}}} \underline{J}(\overrightarrow{\boldsymbol{P}}, \overrightarrow{\boldsymbol{\sigma}}, \overrightarrow{\boldsymbol{W}})$, which is merely a conjecture at present (see Remark 6 ), the inequality in (70) could be derived from the general formula as in the classical case. 
Let us proceed to the proof of the strong converse part

$$
C^{\dagger}\left(\overrightarrow{\boldsymbol{W}} \uparrow_{\overrightarrow{\boldsymbol{c}}, \gamma}\right) \leq \sup _{P \in \mathcal{P}_{c, \gamma}(\mathcal{X})} I(P, W)
$$

under the assumption that $\operatorname{dim} \mathcal{H}<\infty$. We claim that for any $\overrightarrow{\boldsymbol{x}} \in \overrightarrow{\mathcal{X}}=\left\{\hat{\mathcal{X}}^{(n)}\right\}$, where $\hat{\mathcal{X}}^{(n)}=\mathcal{X}_{c^{(n)}, \gamma}^{(n)}$, and any $\sigma \in \mathcal{S}(\mathcal{H})$,

$$
\bar{D}\left(\overrightarrow{\boldsymbol{W}}_{\overrightarrow{\boldsymbol{x}}} \| \overrightarrow{\boldsymbol{\sigma}}\right) \leq \sup _{P \in \mathcal{P}_{c, \gamma}(\mathcal{X})} J(P, \sigma, W),
$$

where $\overrightarrow{\boldsymbol{W}}_{\overrightarrow{\boldsymbol{x}}}=\left\{W_{x^{n}}^{(n)}\right\}$ and $\overrightarrow{\boldsymbol{\sigma}}=\left\{\sigma^{\otimes n}\right\}$. We only need to show this for $\sigma$ such that $\operatorname{Im} \sigma \supset \operatorname{Im} W_{x}$ for $\forall x \in \operatorname{supp}(P), \forall P \in \mathcal{P}_{c, \gamma}(\mathcal{X})$, since the RHS is $\infty$ otherwise. For any $x^{n} \in \hat{\mathcal{X}}^{(n)}$ and any real numbers $a$ and $0 \leq s \leq 1$, it follows from (48) that

$$
\begin{aligned}
\operatorname{Tr}\left[W_{x^{n}}^{(n)}\left\{W_{x^{n}}^{(n)}-e^{n a} \sigma^{\otimes n}>0\right\}\right] & \leq \exp \left[-n\left(\text { as }-\frac{1}{n} \sum_{i=1}^{n} \log \operatorname{Tr}\left[W_{x_{i}}^{1+s} \sigma^{-s}\right]\right)\right] \\
& \leq \exp [-n(a s-\psi(s))],
\end{aligned}
$$

where

$$
\psi(s) \stackrel{\text { def }}{=} \sup _{P \in \mathcal{P}_{c, \gamma}(\mathcal{X})} \sum_{x \in \mathcal{X}} P(x) \log \operatorname{Tr}\left[W_{x}^{1+s} \sigma^{-s}\right] .
$$

Let

$$
\mathcal{P}_{c, \gamma, 2}(\mathcal{X}) \stackrel{\text { def }}{=}\left\{P \mid P \in \mathcal{P}_{c, \gamma}(\mathcal{X}) \text { and }|\operatorname{supp}(P)| \leq 2\right\},
$$

where $|\operatorname{supp}(P)|$ denotes the number of elements of the support of $P$. Then a similar argument to section IV of [18] is applied to prove that $\mathcal{P}_{c, \gamma}(\mathcal{X})$ is the convex hull of $\mathcal{P}_{c, \gamma, 2}(\mathcal{X})$; see Appendix II. Hence we have

$$
\begin{aligned}
\psi(s) & =\sup _{P \in \mathcal{P}_{c, \gamma, 2}(\mathcal{X})} \sum_{x \in \mathcal{X}} P(x) \log \operatorname{Tr}\left[W_{x}^{1+s} \sigma^{-s}\right] . \\
& =\max _{\omega \in \bar{\Omega}} g(s, \omega),
\end{aligned}
$$

where $\bar{\Omega}$ is the compact subset of $[0,1] \times \mathcal{S}(\mathcal{H})^{2}$ defined as the closure of

$$
\Omega=\left\{\left(\lambda, W_{x_{1}}, W_{x_{2}}\right) \mid 0 \leq \lambda \leq 1,\left(x_{1}, x_{2}\right) \in \mathcal{X}^{2}, \lambda c\left(x_{1}\right)+(1-\lambda) c\left(x_{2}\right) \leq \gamma\right\},
$$

and

$$
g\left(s,\left(\lambda, \rho_{1}, \rho_{2}\right)\right) \stackrel{\text { def }}{=} \lambda \log \operatorname{Tr}\left[\rho_{1}^{1+s} \sigma^{-s}\right]+(1-\lambda) \log \operatorname{Tr}\left[\rho_{2}^{1+s} \sigma^{-s}\right] .
$$

A similar argument to the derivation of (51) is applied to (73) so that we have

$$
\begin{aligned}
& \lim _{n \rightarrow \infty} \operatorname{Tr}\left[W_{x^{n}}^{(n)}\left\{W_{x^{n}}^{(n)}-e^{n a} \sigma^{\otimes n}>0\right\}\right]=0 \\
& \text { for } \forall a>\max _{\omega \in \bar{\Omega}} \frac{\partial}{\partial s} g(\omega, 0)=\sup _{P \in \mathcal{P}_{c, \gamma}(\mathcal{X})} J(P, \sigma, W),
\end{aligned}
$$


which proves the claim (72). Now the strong converse (171) is derived as follows:

$$
\begin{aligned}
C^{\dagger}\left(\overrightarrow{\boldsymbol{W}} \uparrow_{\overrightarrow{\boldsymbol{c}}, \gamma}\right) & =\max _{\overrightarrow{\boldsymbol{P}} \in \overrightarrow{\mathcal{P}}(\overrightarrow{\mathcal{\mathcal { X }}})} \min _{\overrightarrow{\boldsymbol{\sigma}} \in \overrightarrow{\mathcal{S}}(\overrightarrow{\mathcal{H}})} \bar{J}(\overrightarrow{\boldsymbol{P}}, \overrightarrow{\boldsymbol{\sigma}}, \overrightarrow{\boldsymbol{W}}) \\
& \leq \min _{\overrightarrow{\boldsymbol{\sigma}} \in \overrightarrow{\mathcal{S}}(\overrightarrow{\mathcal{H}})} \max _{\overrightarrow{\boldsymbol{P}} \in \overrightarrow{\mathcal{P}}(\overrightarrow{\hat{\mathcal{X}}})} \bar{J}(\overrightarrow{\boldsymbol{P}}, \overrightarrow{\boldsymbol{\sigma}}, \overrightarrow{\boldsymbol{W}}) \\
& =\min _{\overrightarrow{\boldsymbol{\sigma}} \in \overrightarrow{\mathcal{S}}(\overrightarrow{\mathcal{H}})} \max _{\overrightarrow{\boldsymbol{x}} \in \overrightarrow{\mathcal{X}}} \bar{D}\left(\overrightarrow{\boldsymbol{W}}_{\overrightarrow{\boldsymbol{x}}} \| \overrightarrow{\boldsymbol{\sigma}}\right) \\
& \leq \min _{\sigma \in \mathcal{S}(\mathcal{H})} \max _{\overrightarrow{\boldsymbol{x}} \in \hat{\hat{\mathcal{X}}}} \bar{D}\left(\overrightarrow{\boldsymbol{W}}_{\overrightarrow{\boldsymbol{x}}} \| \overrightarrow{\boldsymbol{\sigma}}\right) \quad \text { with } \quad \overrightarrow{\boldsymbol{\sigma}}=\left\{\sigma^{\otimes n}\right\} \\
& \leq \min _{\sigma \in \mathcal{S}(\mathcal{H})} \sup _{P \in \mathcal{P}_{c, \gamma}(\mathcal{X})} J(P, \sigma, W) \\
& =\sup _{P \in \mathcal{P}_{c, \gamma}(\mathcal{X})} \min _{\sigma \in \mathcal{S}(\mathcal{H})} J(P, \sigma, W)=\sup _{P \in \mathcal{P}_{c, \gamma}(\mathcal{X})} I(P, W),
\end{aligned}
$$

where we have invoked the fact that similar relations to (47) hold for the present situation.

\section{Concluding remarks}

We have obtained a general formula for capacity of classical-quantum channels together with a characterization of the strong converse property by extending the information-spectrum method to the quantum setting. The general results have been applied to stationary memoryless case with or without cost-constraint on inputs, whereby new simple proofs have been given to the corresponding coding theorems. Among many open problems concerning the present work, we would recall here only the following two; one is the problem mentioned in Remark 6 and the other is how to analyze (if possible) asymptotics of the quantum information spectrum directly, not by way of the theory of quantum hypothesis testing. These problems will be important toward further developement of the quantum information-spectrum method.

\section{Acknowledgment}

The authors are grateful to an anonymous referee and Prof. T.S. Han for useful comments on the history of Feinstein's lemma.

\section{Appendix}

\section{Appendix I Proof of Lemma 1}


Let us begin with the attainability of $c \stackrel{\text { def }}{=} \sup _{\vec{f}}[\overrightarrow{\boldsymbol{f}}]_{x}^{-}$. We assume $-\infty<c<\infty$ first. Then for every natural number $k$ there exists $\vec{f}^{(k)}=\left\{f_{n}^{(k)}\right\}_{n=1}^{\infty} \in \overrightarrow{\mathcal{F}}$ such that $\left[\vec{f}^{(k)}\right]_{x}^{-}>c-\frac{1}{k}$. This implies that

$$
\limsup _{n \rightarrow \infty} f_{n}^{(k)}\left(c-\frac{1}{k}\right) \leq x,
$$

and hence there exists $n_{k}$ such that for any $n \geq n_{k}$,

$$
f_{n}^{(k)}\left(c-\frac{1}{k}\right) \leq x+\frac{1}{k}
$$

Let us choose $\left\{n_{k}\right\}$ to satisfy $n_{k}<n_{k+1}(\forall k)$. Then every $n$ uniquely determines a number $k$ such that $n_{k} \leq n<n_{k+1}$, which we denote by $k=k_{n}$. Letting $f_{n}^{*} \stackrel{\text { def }}{=}$ $f_{n}^{\left(k_{n}\right)} \in \mathcal{F}_{n}$ and $\overrightarrow{\boldsymbol{f}}^{*} \stackrel{\text { def }}{=}\left\{f_{n}^{*}\right\}_{n=1}^{\infty} \in \overrightarrow{\mathcal{F}}$, we have

$$
f_{n}^{*}\left(c-\frac{1}{k_{n}}\right) \leq x+\frac{1}{k_{n}} .
$$

This implies that $\limsup _{n \rightarrow \infty} f_{n}^{*}(c-\varepsilon) \leq x$ for any $\varepsilon>0$, and therefore we have $\left[\overrightarrow{\boldsymbol{f}}^{*}\right]_{x}^{-}=c=\sup _{\vec{f}}[\overrightarrow{\boldsymbol{f}}]_{x}^{-}$. Next, let us consider the case when $c=\infty$. Then for every natural number $k$ there exists $\vec{f}^{(k)}=\left\{f_{n}^{(k)}\right\}_{n=1}^{\infty} \in \overrightarrow{\mathcal{F}}$ such that $\left[\vec{f}^{(k)}\right]_{x}^{-}>k$, which implies the existence of a number $n_{k}$ such that for any $n \geq n_{k}$ we have $f_{n}^{(k)}(k) \leq x+\frac{1}{k}$. Then a similar argument to the previous one is applicable to construction of a sequence $\overrightarrow{\boldsymbol{f}}^{*}=\left\{f_{n}^{*}\right\} \in \overrightarrow{\mathcal{F}}$ satisfying $\limsup _{n \rightarrow \infty} f_{n}^{*}(k) \leq x$ for any $k$, and therefore we have $\left[\overrightarrow{\boldsymbol{f}}^{*}\right]_{x}^{-}=\infty=\sup _{\vec{f}}[\overrightarrow{\boldsymbol{f}}]_{x}^{-}$. The remaining case $c=-\infty$ is trivial, since this means that $[\overrightarrow{\boldsymbol{f}}]_{x}^{-}=-\infty$ for all $\overrightarrow{\boldsymbol{f}} \in \overrightarrow{\mathcal{F}}$.

Let us proceed to the attainability of $c \stackrel{\text { def }}{=} \sup _{\vec{f}}[\overrightarrow{\boldsymbol{f}}]_{x}^{+}$, . Assume $-\infty<c<\infty$. Then for every $k$ there exists $\overrightarrow{\boldsymbol{f}}^{(k)}=\left\{f_{n}^{(k)}\right\}_{n=1}^{\infty} \in \overrightarrow{\mathcal{F}}$ such that $\left[\vec{f}^{(k)}\right]_{x}^{+}>c-\frac{1}{k}$. This implies that

$$
\liminf _{n \rightarrow \infty} f_{n}^{(k)}\left(c-\frac{1}{k}\right)<x,
$$

and hence there exists a $\delta_{k}>0$ such that the set

$$
A_{k} \stackrel{\text { def }}{=}\left\{n \mid f_{n}^{(k)}\left(c-\frac{1}{k}\right) \leq x-\delta_{k}\right\}
$$

has infinitely many elements. Let $\left\{B_{k}\right\}_{k=1}^{\infty}$ be a family of subsets $B_{k} \subset A_{k}$ such that $\left|B_{k}\right|=\infty$ and $B_{k} \cap B_{l}=\phi$ for $\forall k \neq \forall l$, and let $\overrightarrow{\boldsymbol{f}}^{*}=\left\{f_{n}^{*}\right\}_{n=1}^{\infty}$ be defined by

$$
f_{n}^{*}=\left\{\begin{array}{cll}
f_{n}^{(k)} & \text { if } & n \in B_{k}, \\
\text { an arbitrary element of } \mathcal{F}_{n} & \text { if } & n \notin \bigcup_{k} B_{k} .
\end{array}\right.
$$


Then for every $k$ the set $\left\{n \mid f_{n}^{*}\left(c-\frac{1}{k}\right) \leq x-\delta_{k}\right\}$ includes $B_{k}$ as a subset and hence has infinitely many elements. This leads to $\liminf _{n \rightarrow \infty} f_{n}^{*}(c-\varepsilon)<x$ for any $\varepsilon>0$, and therefore we have $\left[\overrightarrow{\boldsymbol{f}}^{*}\right]_{x}^{+}=c=\sup _{\overrightarrow{\boldsymbol{f}}}[\overrightarrow{\boldsymbol{f}}]_{x}^{+}$. The case $c=\infty$ can be proved similarly, and the case $c=-\infty$ is trivial.

Letting $\mathcal{G}_{n}$ be the set of monotonically nondecreasing functions $g_{n}(a) \stackrel{\text { def }}{=}-f_{n}(-a)$ for $f_{n} \in \mathcal{F}_{n}$, we have

$$
\inf _{\overrightarrow{\boldsymbol{f}} \in \overrightarrow{\mathcal{F}}}[\overrightarrow{\boldsymbol{f}}]_{x}^{-}=-\sup _{\vec{g} \in \overrightarrow{\mathcal{G}}}[\vec{g}]_{-x}^{+} \quad \text { and } \quad \inf _{\overrightarrow{\boldsymbol{f}} \in \overrightarrow{\mathcal{F}}}[\overrightarrow{\boldsymbol{f}}]_{x}^{+}=-\sup _{\vec{g} \in \overrightarrow{\mathcal{G}}}[\vec{g}]_{-x}^{-}
$$

The attainability of the infimums thus follows from that of the supremums.

\section{Appendix II Proof that $\mathcal{P}_{c, \gamma}(\mathcal{X})$ is the convex hull of $\mathcal{P}_{c, \gamma, 2}(\mathcal{X})$}

Let $P$ be an arbitrary distribution in $\mathcal{P}_{c, \gamma}(\mathcal{X})$, and let $\mathcal{R}_{P}$ denote the subset of $\mathcal{P}_{c, \gamma}(\mathcal{X})$ consisting of all distributions $P^{\prime}$ satisfying $E_{P^{\prime}}[c]=E_{P}[c]$ and $\operatorname{supp}\left(P^{\prime}\right) \subset$ $\operatorname{supp}(P)$. Since $\mathcal{R}_{P}$ is convex and compact, the element $P$ of $\mathcal{R}_{P}$ can be represented as a convex combination of extreme points of $\mathcal{R}_{P}$. Hence it suffices to show that the support of every extreme point of $\mathcal{R}_{P}$ has at most two elements. Suppose that a $P^{\prime} \in \mathcal{R}_{P}$ is written as $P^{\prime}=\sum_{i=1}^{k} \lambda_{i} \delta_{x_{i}}$, where $\left\{x_{1}, \ldots, x_{k}\right\}=\operatorname{supp}\left(P^{\prime}\right)$ and $\lambda_{i} \stackrel{\text { def }}{=} P^{\prime}\left(x_{i}\right)>0$. If $k \geq 3$, there exists a nonzero real vector $\left(\alpha_{1}, \ldots, \alpha_{k}\right) \in \mathbb{R}^{k}$ such that $\sum_{i=1}^{k} \alpha_{i}=0$ and $\sum_{i=1}^{k} \alpha_{i} c\left(x_{i}\right)=0$. Then, for a sufficiently small $\varepsilon>0$, $P_{1}=\sum_{i=1}^{k}\left(\lambda+\varepsilon \alpha_{i}\right) \delta_{x_{i}}$ and $P_{2}=\sum_{i=1}^{k}\left(\lambda-\varepsilon \alpha_{i}\right) \delta_{x_{i}}$ become two distinct distributions in $\mathcal{R}_{P}$ and satisfy $P^{\prime}=\frac{1}{2}\left(P_{1}+P_{2}\right)$, which means that $P^{\prime}$ is not extreme. Therefore, if $P^{\prime}$ is an extreme point then $k=\left|\operatorname{supp}\left(P^{\prime}\right)\right| \leq 2$.

\section{References}

[1] A.S. Holevo, "The capacity of the quantum channel with general signal states," IEEE Trans. Inform. Theory, vol.44, 269-273, 1998.

[2] B. Schumacher and M.D. Westmoreland, "Sending classical information via noisy quantum channels," Phys. Rev. A, vol.56, 131-138, 1997.

[3] A.S. Holevo, "Bounds for the quantity of information transmitted by a quantum communication channel," Probl. Inform. Transm., vol.9, 177-183, 1973.

[4] A.S. Holevo, "On the capacity of quantum communication channel," Probl. Inform. Transm., vol. 15, no. 4, pp. 247-253, 1979.

[5] S. Verdú and T.S. Han, "A general formula for channel capacity," IEEE Trans. Inform. Theory, vol.40, 1147-1157, 1994. 
[6] T.S. Han, Information-Spectrum Methods in Information Theory, SpringerVerlag, 2003. (The original Japanese edition was published from Baifukan-Press, Tokyo, in 1998.)

[7] A. Winter, "Coding theorem and strong converse for quantum channels," IEEE Trans. Inform. Theory, vol.45, 2481-2485, 1999.

[8] A. S. Holevo, "An analog of the theory of statistical decisions in noncommutative theory of probability," Trudy Moskov. Mat. Ob̌̌č., vol. 26, 133-149 1972. (English translation is Trans. Moscow Math. Soc., vol. 26, 133-149 1972.)

[9] C.W. Helstrom, Quantum Detection and Estimation Theory, Academic Press, New York, 1976.

[10] H. Nagaoka and M. Hayashi, "An information-spectrum approach to classical and quantum hypothesis testing," LANL e-print quant-ph/0206185, 2002.

[11] F. Hiai and D. Petz, "The proper formula for relative entropy and its asymptotics in quantum probability," Commun. Math. Phys., vol. 143, 99-114, 1991.

[12] T. Ogawa and H. Nagaoka, "Strong converse and Stein's lemma in quantum hypothesis testing," IEEE Trans. Inform. Theory, vol.46, 2428-2433, 2000. LANL e-print quant-ph/9906090, 1999.

[13] T. Ogawa, "A study on the asymptotic property of the hypothesis testing and the channel coding in quantum mechanical systems," Ph.D. dissertation, University of Electro-Communications, 2000 (In Japanese).

[14] T. Ogawa and H. Nagaoka, "A New Proof of the Channel Coding Theorem via Hypothesis Testing in Quantum Information Theory," Proc. 2002 IEEE International Symposium on Information Theory, p.73. 2002.

[15] T. Ogawa and H. Nagaoka, "Strong Converse to the Quantum Channel Coding Theorem," IEEE Trans. Inform. Theory, vol.45, 2486-2489, 1999.

[16] A.S. Holevo, "Coding theorems for quantum channels," LANL e-print quant-ph/9809023, 1998.

[17] A.S. Holevo, "Problems in the mathematical theory of quantum communication channels," Rep. Math. Phys., vol.12, no.2, pp.273-278, 1977.

[18] A. Fujiwara and H. Nagaoka, "Operational capacity and pseudoclassicality of a quantum channel," IEEE Trans. Inform. Theory, vol.44, 1071-1086, 1998.

[19] C.E. Shannon, "Certain results in coding theory for noisy channels," Information and Control vol.1, 6-25, 1957. 
[20] D. Blackwell, L. Breiman and A.J. Thomasian, "The capacity of a class of channels," Ann. Math. Statist, vol.30, 1229-1241, 1959.

[21] A. Feinstein, "A new basic theorem of information theory," IRE Trans. PGIT, vol.4, 2-22, 1954.

[22] S. Osawa and H. Nagaoka, "Numerical experiments on the capacity of quantum channel with entangled input states," IEICE Trans., vol.E84-A, 2583-2590, 2001.

[23] P.W. Shor, "Additivity of the classical capacity of entanglement-breaking quantum channels," LANL e-print quant-ph/0201149, 2002.

[24] C. King, "Additivity for a class of unital qubit channels," LANL eprint quant-ph/0103156, 2001 (Jour. Math. Phys., in press).

[25] C. King, "The capacity of the quantum depolarizing channel," LANL eprint quant-ph/0204172, 2002.

[26] A. Uhlmann, "Entropy and Optimal Decompositions of States Relative to a Maximal Commutative Subalgebra," Open Systems $\&$ Information Dynamics, vol.5, 209-228, 1998.

[27] T. Ogawa and M. Hayashi, "On error exponents in quantum hypothesis testing," LANL e-print quant-ph/0206151, 2002.

[28] M. Ohya, D. Petz and N. Watanabe, "On capacities of quantum channels," Prb. Math. Stat., vol.17, 179-196, 1997.

[29] B. Schumacher and M.D. Westmoreland, "Optimal signal ensembles," Phys. Rev. A, vol 63, no.2, 022308, Jan. 2001.

[30] I. Csiszár and J. Körner, Information Theory: Coding Theorems for Discrete Memoryless Systems, Academic Press, 1981.

[31] R. G. Gallager, Information Theory and Reliable Communication, John Wiley \& Sons, 1968.

[32] I. Ekeland and R. Téman, Convex Analysys and Variational Problems, NorthHolland, 1976; SIAM, 1999.

[33] A. S. Holevo, "On quantum communication channels with constrained inputs," LANL e-print quant-ph/9705054, 1997. 